\title{
Light dark matter candidates in intense laser pulses I: paraphotons and fermionic minicharged particles
}

\section{S. Villalba-Chávez and C. Müller}

Institut für Theoretische Physik I, Heinrich Heine Universität Düsseldorf, Universitätsstr. 1, 40225 Düsseldorf, Germany

E-mail: selym@tp1.uni-duesseldorf.de, c.mueller@tp1.uni-duesseldorf.de

ABSTRACT: Polarimetric experiments driven by the strong field of a circularly polarized laser wave can become a powerful tool to limit the parameter space of not yet detected hidden-photons and minicharged particles associated with extra U(1) gauge symmetries. We show how the absorption and dispersion of probe electromagnetic waves in the vacuum polarized by such a background are modified due to the coupling between the visible U(1)gauge sector and these hypothetical degrees of freedom. The results of this analysis reveal that the regime close to the two-photon reaction threshold can be a sensititive probe of these hidden particles. Parameters of modern laser systems are used to estimate the projected sensitivities on the corresponding coupling constants in regions where experiments driven by dipole magnets are less constricted. The role played by a paraphoton field is analyzed via a comparison with a model in which the existence of minicharges is assumed only. For both scenarios is found that the most stringent exclusion limit occurs at the lowest threshold mass; this one being determined by a certain combination of the field frequencies and dictated by energy momentum balance of the photo-production of a pair of minicharged particles. The dependencies of the observables on the laser attributes as well as on the unknown particle parameters are also analyzed.

KeYwords: Beyond Standard Model, Electromagnetic Processes and Properties

ARXIV EPRINT: 1412.4678 


\section{Contents}

1 Introduction 1

2 Loop-induced photon-paraphoton oscillations $\quad 4$

2.1 Kinetic mixing and effective action 4

$\begin{array}{lll}2.2 & \text { Absorption and dispersion of small-amplitude waves } & 7\end{array}$

$\begin{array}{lll}2.3 & \text { Spectral decomposition at } \xi_{\epsilon}<1 & 10\end{array}$

$\begin{array}{lll}2.4 & \text { Conversion probability and polarimetric observables } & 12\end{array}$

3 Experimental prospects $\quad 14$

$\begin{array}{lll}3.1 & \text { Estimating the exclusion limits } & 14\end{array}$

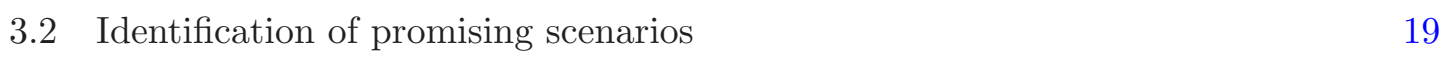

4 Conclusions $\quad 23$

\section{Introduction}

String theory encompasses at present some of the most promising candidates for a unified description of the fundamental forces in nature. Four-dimensional remnants resulting from the compactification of extra dimensions, provide a variety of Standard Model (SM) extensions which very often contain - in addition to the $\mathrm{SU}(3) \times \mathrm{SU}(2) \times \mathrm{U}(1)$ gauge group - a hitherto unobserved very weakly interacting sector involving further gauge invariances [14]. The derivation of effective theories with such symmetry properties opens a portal for the insertion of hidden degrees of freedom whose realization in nature might be linked to the abundant dark matter and dark energy of our universe [5-8]. Determining the extent to which these outcomes adjust to a realistic description of the latter subject is a fundamental task in particle physics. Mainly, because it could not only reveal why other puzzles in the SM lack a satisfactory theoretical explanation but could also validate the building blocks on which it relies. Notably, with respect to the charge quantization, as of today it is still not clear whether or not it represents a fundamental principle. Indeed, the conclusion resulting from some effective scenarios is that this might not be the case once the hidden sector includes an extra U(1) symmetry and the respective paraphoton [9-12] or hiddenphoton field - minimally coupled to very light particles under the same $\mathrm{U}(1)$ group - is kinetimatically mixed with the visible electromagnetic sector. It is precisely the diagonalization of this term what allows us to predict carriers with unquantized electric charges. In this context, a presumably feeble interaction leads to introduce Mini-Charged Particles (MCPs) [13-16], a concept which often arises in many branches beyond the SM [17-19].

Quantum Electrodynamics (QED) ammended with the incorporation of the hiddenphoton field and very light MCPs, acquires a source of quantum fluctuations which could 
induce nonlinear interactions in the electromagnetic field as those mediated by virtual electron-positron pairs [20,21]. Because of this fact, its phenomenology can be modified and experiments in strong-external fields - searching for elusive phenomena such as birefringence and dichroism of the vacuum [22-24], discrepancies in the Coulomb law [25, 26] or the generation of visible photons from these hypothetical degrees of freedom $[27,28]-$ can become illuminating tools for testing their occurence in nature. Inspired by this fact, several laboratory-based experiments searching for signatures of MCPs, paraphotons and axionlike particles have been carried out. Indeed, on the basis of the mentioned optical properties of the polarized vacuum, collaborations such as BFRT [29], PVLAS [30, 31], BMV [32], and Q \& A [33] have performed high-precision polarimetric measurements on a low-energy photon beam which traverses a magnetic field region. Likewise, based on the idea of photon regeneration, many "Light Shining Through a Wall" experiments have been put forward [34-42], but in none of these setups a weakly interacting sub-eV particle has been detected so far. Instead, the range of the unknown particle masses and coupling constants has been constricted.

Currently, the record for the most stringent bounds on MCPs parameters [e. g., relative charge parameter $\epsilon \lesssim 10^{-14}$ for masses below a few $\mathrm{keV}$ ] result from arguments related to stellar cooling [43] which are not observed in Horizontal Branch stars. This exclusion limit is, nonetheless, somewhat arguable since the inclusion of macroscopic parameters such as the density and the temperature of the star might attenuate it significantly [44, 45]. Such observations motivate the interest in laboratory searches, ideally, with enough sensitivity as to compete with the astrophysical bounds. However, due to technical limitations, the laboratory tests via polarimetry and "Light Shining Through a Wall" do not yet reach this goal. The main difficulty stems from a presumably feeble coupling between these particles and the magnetic field $|\boldsymbol{B}| \lesssim 10^{5} \mathrm{G}$, which can be effectively extended up to distances on the order of $L \sim 1 \mathrm{~km}$ by using high-finesse interferometry. So, to make manifest the existence of such degrees of freedom, a significant improvement in the field strength as well as in the mentioned techniques are required. Meanwhile, the upper limits derived from the outcomes of the cited experimental collaborations remain many orders of magnitude bigger than the astrophysical one. While a new generation of "Light Shining Through a Wall" experiments might overcome this obstacle [46-48], there is a real demand for new theoretical efforts toward the search of complementary scenarios where potential improvements in the bounds of parameters of these dark matter candidates can be achieved [49-51].

Despite the disadvantage introduced by their limited temporal and spatial extensions, the prospect of finding stringent limits on the weakly interacting sub-eV particles' attributes by using high-intensity laser fields is becoming a subject of interest [52-58]. Firstly, because the field strengths attained from these powerful sources are much higher than the static ones frequently used in experiments driven by dipole magnets $|\boldsymbol{B}| \sim o\left[10^{4}-10^{5}\right] \mathrm{G}$; and secondly, because the oscillating nature of laser fields introduces - apart from the profile of the wave amplitude and its polarization - the field frequency as an additional characteristic. As a consequence, the processes occuring inside these kind of backgrounds are typified by thresholds and resonances related to the exchange of energy between the quantum fields 
and the classical laser wave. In connection, a diversity of processes involving a frequency shift of probe beams are predicted to occur leading to introduce novel detection techniques such as the Raman spectroscopy [53, 57]. Indeed, some phenomenological studies in this direction are pointing out that the nonobservation of these inelastic scattering waves could allow us to limit the parameter space of axionlike particles and MCPs in regions for which the current laboratory-based experiments establish less stringent sensitivities.

Clearly, investigations of this nature are also stimulated by ongoing projects such as the Extreme Light Infrastructure (ELI) [59] and the Exawatt Center for Extreme Light Studies (XCELS) [60]. The — so far inaccessible - field strengths to be attained in these high-intensity laser facilities $|\boldsymbol{B}| \sim o\left[10^{11}-10^{12}\right]$ G offer a genuine opportunity for studying the low energy sector of particle physics as well as for observing - among other hitherto undetected nonlinear QED phenomena [61-66] — the spontaneous production of electronpositron pairs [67-69]. While this constitutes a very strong motivation, the first estimates of the upper bounds resulting from operating facilities such as the Petawatt High-Energy Laser for heavy Ion eXperiments (PHELIX) [70] and the Laboratoire pour l'Utilisation des Lasers Intenses (LULI) [71] might turn out to be competitive in the search for MCPs and even more promising than those derived from ELI and XCELS parameters. This has already been predicted theoretically for axionlike particles [58]. The main reason behind this finding lies in the fact that these contemporary systems - although operating at moderate intensity $I \sim o\left[10^{14}-10^{16}\right] \mathrm{W} / \mathrm{cm}^{2}$ - deliver relatively long pulses $\tau \sim o[\mathrm{~ns}]$. For axionlike particles the relevant combination $I \tau^{2} \sim o\left[10^{14}-10^{16}\right] \mathrm{W} \mathrm{ns}{ }^{2} / \mathrm{cm}^{2}$ increases the sensitivity in polarimetric experiments when compared with the outcomes resulting from the ELI and XCELS parameters at which the temporal lengths $\tau \sim o[\mathrm{fs}]$ significantly compensate the beneficial aspects introduced by the expected high intensities $I \sim o\left[10^{25}-10^{26}\right] \mathrm{W} / \mathrm{cm}^{2}$ $\left[I \tau^{2} \sim o\left[10^{13}-10^{14}\right] \mathrm{W} \mathrm{ns}^{2} / \mathrm{cm}^{2}\right]$

Against this background, the present work aims to provide a first estimate on the exclusion limits for MCPs and massless paraphotons, resulting from plausible polarimetric setups utilizing the field of a circularly polarized laser wave of long temporal length. To this end, we first determine how the vacuum refraction indices and the photon absorption coefficients that follow from the vacuum polarization tensor mediated by fermionic MCP pairs are modified by a hidden-photon field. We find that the birefringence and dichroism of the vacuum are quite pronounced in a vicinity of the first photo-production threshold. Our analysis reveals that - at moderate laser intensities — high-precision polarimetric experiments might be sensitive probes of these hidden degrees of freedom. Parameters of the aforementioned laser facilities are used for establishing upper bounds on the respective parameter spaces. The role played by a paraphoton field is analyzed via a comparison with a model in which the existence of MCPs only is assumed. For both scenarios is found that the most stringent exclusion limit occurs at the lowest threshold mass; this one being determined by a certain combination of the laser frequencies and dictated by energy momentum balance of the photo-production of a pair of minicharged particles. An analysis of the signal's dependence on the laser attributes as well as the on the unknown parameters is also included. 


\section{Loop-induced photon-paraphoton oscillations}

\subsection{Kinetic mixing and effective action}

So far there are no experimental evidences which indicate a violation of any fundamental principle of QED. Hence, we will consider the most simple renormalizable Lagrangian density that includes both an electromagnetic field $a_{\mu}(x)$ and a hidden vector field $w_{\mu}(x)$ but preserves the Lorentz invariance, the spatial parity, the temporal reversibility and the charge conjugation symmetry. Furthermore, we wish to guarantee the gauge invariance of the involved fields and avoid the proliferation of an additional charge labeling the elementary standard-model particles. In order to satisfy these two conditions, we deal with a theory invariant under a $\mathrm{U}(1) \times \mathrm{U}(1)$-gauge symmetry group and assume that the interaction between both Abelian sectors occurs through a kinetic-mixing term characterized by a completely arbitrary dimensionless parameter $\chi_{0}$. With these details in mind, the Lagrangian density turns out to be $[5-8]^{1}$

$$
\mathcal{L}=-\frac{1}{16 \pi} f_{\mu \nu} f^{\mu \nu}-\frac{1}{16 \pi} h_{\mu \nu} h^{\mu \nu}-\frac{1}{8 \pi} \chi_{0} f_{\mu \nu} h^{\mu \nu}+e_{h} j_{h}^{\mu} w_{\mu},
$$

where $f_{\mu \nu}=\partial_{\mu} a_{\nu}-\partial_{\nu} a_{\mu}$ and $h_{\mu \nu}=\partial_{\mu} w_{\nu}-\partial_{\nu} w_{\mu}$ refer to the corresponding field tensors; $j_{h}^{\mu}$ and $e_{h}$ is the hidden current and gauge coupling respectively associated with hypothetical particles charged under the extra U(1) symmetry. The explicitly expression of $j_{h}^{\mu}$ depends on the nature of the hidden matter sector. Hereafter we suppose that it is determined by Dirac fermions. The kinetic mixing in eq. (2.1) can be diagonalized by changing the hidden gauge field to another basis $w_{\mu} \rightarrow w_{\mu}-\chi_{0} a_{\mu}$. After having used the sequence of redefinitions $a_{\mu} \rightarrow\left(1-\chi_{0}^{2}\right)^{-1 / 2} a_{\mu}$ and $\chi_{0} \rightarrow \chi\left(1-\chi_{0}^{2}\right)^{1 / 2}$ we end up with

$$
\mathcal{L}=-\frac{1}{16 \pi} f_{\mu \nu} f^{\mu \nu}-\frac{1}{16 \pi} h_{\mu \nu} h^{\mu \nu}+e_{h} j_{h}^{\mu} w_{\mu}-\chi e_{h} j_{h}^{\mu} a_{\mu} .
$$

Manifestly, the last term defines an interacting vertex which links the hidden matter sector and the electromagnetic field. As a consequence, the hypothetical particles acquire an electric charge under the visible U(1)-gauge field given by

$$
q_{\epsilon} \equiv \epsilon e=-\chi e_{h} .
$$

Since $\chi$ is an arbitrary number, the parameter $\epsilon$ - which acccounts for the potentially small coupling strengh in units of the electron charge $e$ - is not necessarily an integer number. For small values of $\chi \ll 1$, one finds that $|\epsilon| \ll 1$ due to which the weakly interacting charge carriers are called MCPs.

It is opportune to emphasize that the hidden-photons can, in general, acquire a mass term $\sim m_{\gamma^{\prime}}^{2} w_{\mu} w^{\mu}$ through the Higgs mechanism leading to a break down of the initial hidden $\mathrm{U}(1)$-symmetry [5-8]. With the change of basis that brings the kinetic-mixing term to a diagonal form, the visible photons become massive particles $\sim \chi^{2} m_{\gamma^{\prime}}^{2} a_{\mu} a^{\mu}$ and the resulting Lagrangian density $\mathcal{L}$ is no longer gauge invariant. The aforementioned transformation

\footnotetext{
${ }^{1}$ From now on "natural" and Gaussian units with $c=\hbar=4 \pi \epsilon_{0}=1$ are used.
} 
creates, in addition, a massive-mixing term $\sim-\chi m_{\gamma^{\prime}}^{2} a_{\mu} w^{\mu}$ which can drive the photonparaphoton oscillations. However, we are motivated to investigate such a phenomenon mediated by a loop diagram of MCPs rather than the previous tree level case. To this end we will suppose that the loop-contributions are dominant in the conversion process as well as in the dispersion relations. In such a situation, the hidden mass term can be ignored, the original $\mathrm{U}(1) \times \mathrm{U}(1)$-symmetry is preserved and the Lagrangian density in eq. (2.2) becomes the starting point of further considerations.

We shall assume throughout that the charged particles involved in eq. (2.2) are coupled to a circularly polarized monochromatic plane-wave

$$
\mathscr{A}^{\mu}(x)=a_{1}^{\mu} \cos (\varkappa x)+a_{2}^{\mu} \sin (\varkappa x)
$$

by the standard minimal-coupling scheme so that the following Lagrangian density

$$
\mathcal{L}_{\text {ext }}=-\chi e_{h} j_{h}^{\mu} \mathscr{A}_{\mu}
$$

must be added to $\mathcal{L}$ [eq. (2.2)]. The external field in eq. (2.4) is chosen in the Lorenz gauge $\partial \mathscr{A}=0$. Therefore, the wave four-vector $\varkappa^{\mu}=\left(\varkappa^{0}, \varkappa\right)$ and the constant vectors along the polarization directions $a_{i}^{\mu}$ (with $i=1,2$ ) satisfy the relations $\varkappa a_{i}=0, \varkappa^{2}=0$, and $a_{1}^{2}=a_{2}^{2} \equiv a^{2}$.

The equations of motion that follow from the combination of eqs. (2.2) and (2.5) can be used to determine the effective action as it follows from a Legendre transform of the generating functional of the connected Green's functions. The integro-differential ansatz which allows us to reach this aim is known in the literature [72-75]. Its application to the problem under consideration leads to express the gauge sector of the generating functional of one-particle irreducible Feynman graphs in the following form

$$
\Gamma[\boldsymbol{\Phi}]=\frac{1}{2} \int d^{4} x d^{4} x^{\prime} \boldsymbol{\Phi}^{\mathrm{T}}(x) \mathcal{D}^{-1}\left(x, x^{\prime}\right) \boldsymbol{\Phi}\left(x^{\prime}\right)+\ldots,
$$

where the abbreviation $+\ldots$ stands for higher order terms in the small-amplitude gauge fields. The inverse Green's function $\mathcal{D}^{-1}\left(x, x^{\prime}\right)$ and the flavor field $\boldsymbol{\Phi}(x)$ involved in eq. (2.6) are given by

$$
\mathcal{D}^{-1}\left(x, x^{\prime}\right) \equiv\left[\begin{array}{cc}
\mathscr{D}_{\mu \nu ; a}^{-1}\left(x, x^{\prime}\right) & \frac{1}{4 \pi} \Pi_{\mu \nu ; o}\left(x, x^{\prime}\right) \\
\frac{1}{4 \pi} \Pi_{\mu \nu ; o}\left(x, x^{\prime}\right) & \mathscr{D}_{\mu \nu ; w}^{-1}\left(x, x^{\prime}\right)
\end{array}\right] \quad \text { and } \quad \boldsymbol{\Phi}(x)=\left[\begin{array}{c}
a_{\mu}(x) \\
w_{\mu}(x)
\end{array}\right],
$$

respectively. The diagonal components in $\mathcal{D}^{-1}\left(x, x^{\prime}\right)$ are the respective two-point proper correlation functions associated with the fields $a_{\mu}(x)$ and $w_{\mu}(x)$. Explicitly,

$$
\mathscr{D}_{\mu \nu ; i}^{-1}\left(x, x^{\prime}\right)=\frac{1}{4 \pi}\left(\square g_{\mu \nu}-\partial_{\mu} \partial_{\nu}\right) \delta^{4}\left(x-x^{\prime}\right)+\frac{1}{4 \pi} \Pi_{\mu \nu ; i}\left(x, x^{\prime}\right), \quad i=a, w
$$

where $\square \equiv \partial_{\mu} \partial^{\mu}=\partial^{2} / \partial t^{2}-\nabla^{2}$ and the metric tensor reads $g_{\mu \nu}=\operatorname{diag}(+1,-1,-1,-1)$. The off-diagonal terms in eq. (2.7) $\frac{1}{4 \pi} \Pi_{\mu \nu ; o}\left(x, x^{\prime}\right)$ and the last contribution embedded in the expression above, i. e., $\frac{1}{4 \pi} \Pi_{\mu \nu ; i}\left(x, x^{\prime}\right)$ with $i=a, w$ enconde the analytic structures of the 

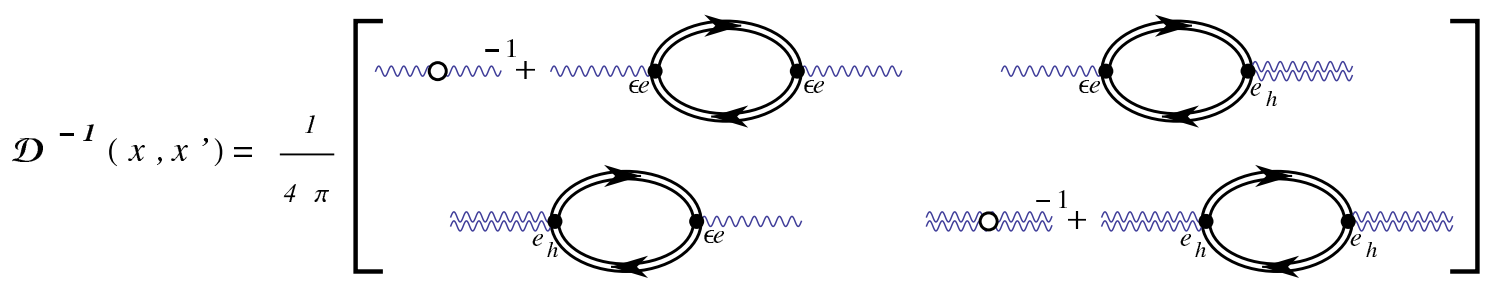

Figure 1. Pictorial representation of the inverse Green's function $\mathcal{D}^{-1}\left(x, x^{\prime}\right)$ in the one-loop approximation. The double lines represent the propagator of MCPs including the full interaction with the external field. A single wavy line denotes the amputated leg corresponding to a small-amplitude electromagnetic wave. Conversely, a double wavy line refers to the amputated leg associated with a hidden-photon field. The unperturbated propagators - inverses of the leading order operators in the right-hand-side of eq. (2.8) - are indicated by open blobs.

two-point irreducible Feynman diagrams. While the latter terms are responsible for pure scattering processes ${ }^{2}$ the former drives the photon-paraphoton oscillations. In such a case, the physical propagating modes are certain mixtures of photon and hidden-photon states resulting from the diagonalization of $\boldsymbol{D}^{-1}\left(x, x^{\prime}\right)$. The described forms of interactions are determined by the causal Feynman propagator of the minicharged carriers in the external field [Furry picture]. Because of this fact, a dependence on the strong electromagnetic background is introduced.

Obviously, in the one-loop approximation, the dressed vertices are reduced to those involved in the initial Lagrangian density [eq. (2.2)]. As a consequence, the inverse Green's function $\mathcal{D}^{-1}\left(x, x^{\prime}\right)$ acquires a simple diagramatic representation [see figure 1] and the tensors driving the photon-paraphoton oscillations and the respective paraphoton scattering process become proportional to $\Pi_{\mu \nu ; a}\left(x, x^{\prime}\right) \equiv \Pi_{\mu \nu}\left(x, x^{\prime}\right)$. Explicitly,

$$
\Pi_{\mu \nu ; o}\left(x, x^{\prime}\right)=-\frac{1}{\chi} \Pi_{\mu \nu}\left(x, x^{\prime}\right) \quad \text { and } \quad \Pi_{\mu \nu ; w}\left(x, x^{\prime}\right)=\frac{1}{\chi^{2}} \Pi_{\mu \nu}\left(x, x^{\prime}\right),
$$

where eq. (2.3) has been used. The analytical properties of $\Pi_{\mu \nu}\left(x, x^{\prime}\right)$ do not differ from the vacuum polarization tensor that arises in a pure QED context. Hence, an appropriate replacement of the electron parameters $(e, m)$ by the respective quantities associated with an $\operatorname{MCP}\left(q_{\epsilon}, m_{\epsilon}\right)$ is enough for acquiring the necessary insights on the structural nature of $\Pi_{\mu \nu}\left(x, x^{\prime}\right)$, and in the related forms of interactions [eq. (2.9)].

We conclude this subsection by obtaining the Dyson-Schwinger equations $[72,74,75]$ from the quadratic part of our effective action [eq. (2.6)]. In momentum space they read

$$
\begin{gathered}
k^{2} a_{\mu}(k)-\int \frac{d^{4} k^{\prime}}{(2 \pi)^{4}} \Pi_{\mu \nu}\left(k, k^{\prime}\right) a^{\nu}\left(k^{\prime}\right)+\frac{1}{\chi} \int \frac{d^{4} k^{\prime}}{(2 \pi)^{4}} \Pi_{\mu \nu}\left(k, k^{\prime}\right) w^{\nu}\left(k^{\prime}\right)=0, \\
k^{2} w_{\mu}(k)-\frac{1}{\chi^{2}} \int \frac{d^{4} k^{\prime}}{(2 \pi)^{4}} \Pi_{\mu \nu}\left(k, k^{\prime}\right) w^{\nu}\left(k^{\prime}\right)+\frac{1}{\chi} \int \frac{d^{4} k^{\prime}}{(2 \pi)^{4}} \Pi_{\mu \nu}\left(k, k^{\prime}\right) a^{\nu}\left(k^{\prime}\right)=0,
\end{gathered}
$$

provided that both Abelian fields are chosen in the Lorenz gauge $k^{\mu} a_{\mu}=0, k^{\mu} w_{\mu}=0$. Note that so far, we have not made use of the precise form of the external wave [eq. (2.4)]

\footnotetext{
${ }^{2}$ For instance, $\Pi_{\mu \nu ; a}$ contributes to photon-photon scattering.
} 
and therefore, the formulae above apply whatever be the nature of the background electromagnetic field. Furthermore, by ignoring the terms proportional to $\sim 1 / \chi$, one can analyze a model in which MCPs exist without the occurence of both: the kinetic-mixing term and the hidden-photon field. Our study intends to establish comparisons between this pure MCPs scenario and the full model described by eq. (2.10) and (2.11).

\subsection{Absorption and dispersion of small-amplitude waves}

In the field of a circularly polarized wave [eq. (2.4)], the polarization tensor in momentum space splits into two relevant terms:

$$
\Pi^{\mu \nu}\left(k, k^{\prime}\right)=(2 \pi)^{4} \delta^{4}\left(k-k^{\prime}\right) \Pi_{0}^{\mu \nu}\left(k^{\prime}\right)+\sum_{j=+,-}(2 \pi)^{4} \delta^{4}\left(k-k^{\prime}+2 j \varkappa\right) \Pi_{j}^{\mu \nu}\left(k^{\prime}\right)
$$

out of which the inelastic contribution - second term in eq. (2.12) - describes scattering processes characterized by the simultaneous emission or absorption of photons of the highintensity laser wave upon the scattering event. The precise structure of $\Pi_{ \pm}^{\mu \nu}\left(k^{\prime}\right)$ is not relevant for what follows. However, the part responsible for the elastic process - first term in eq. (2.12) - deserves to be explained in some detail. This contribution is diagonalizable by using a vector basis that manifests the underlying invariance properties of the vacuum

$$
\Pi_{0}^{\mu \nu}(k)=-\sum_{i=+,-} \pi_{i} \Lambda_{i}^{\mu} \Lambda_{i}^{\nu *}
$$

because the two relevant contributions are determined by transverse eigenstates of opposite helicities $k_{\mu} \Lambda_{ \pm}^{\mu}=0$, subject to the normalization conditions $\Lambda_{+} \Lambda_{-}=-1$ with $\Lambda_{ \pm} \Lambda_{ \pm}=0$ and $\Lambda_{ \pm}^{*}=\Lambda_{\mp}$. Formally, there should occur two additional terms in the diagonal expansion in eq. (2.13). One of these ommited contributions turns out to be longitudinal by construction $\sim k^{\mu} k^{\nu}$, but owing to the gauge invariance property of the polarization tensor $\Pi_{0}^{\mu \nu} k_{\nu}=0$, its corresponding eigenvalue vanishes identically. It is worth mentioning that the previous statement is independent of any approximation used in the calculation of $\Pi_{\mu \nu}$. The remaining disregarded term is originally proportional to a transverse four-vector $\Lambda_{3}^{\mu} \sim \varkappa^{\mu} k^{2}-k^{\mu}(k \varkappa)$, its eigenvalue being proportional to $k^{2}$ in the one-loop approximation. The latter leads to a trivial dispersion equation $k^{2}=0$ in which case $\Lambda_{3}^{\mu} \sim k_{\mu}$ becomes a longitudinal vector that cannot be associated with a physical propagating mode [57, 76, 79].

The relevant eigenvalues $\pi_{ \pm}=-\left(\alpha_{3} \pm i \alpha_{1}\right)\left[\Pi_{0 \nu}^{\mu} \Lambda_{ \pm}^{\nu}=\pi_{ \pm} \Lambda_{ \pm}^{\mu}\right]$ are unwieldy complex functions determined by the form factors $\alpha_{1,3}$ introduced by Bă̌er, Mil'shter̆n and Strakhovenko in ref. [76]. Accordingly, they are represented as twofold parametric integrals which, in general, cannot be evaluated analytically. In fact, when the polarization effects are tiny corrections to the free photon dispersion equation $\left[k^{2}=w^{2}-k^{2} \simeq 0\right]$, the inelastic contributions are strongly suppressed by energy-momentum conservation ${ }^{3}$ and one

\footnotetext{
${ }^{3}$ The partial production rates associated with the generation of an inelastic scattered wave accompanied by a flip of polarization can be read off from the modulus square of the amplitudes that follow from the last term in eq. (2.12) after integration over the final momentum space. Because of the associated Dirac deltas $\delta^{4}\left(k-k^{\prime} \pm 2 \varkappa\right)$, the last operation leads to partial rates proportional to $\propto \delta\left(|\boldsymbol{k}|-|\boldsymbol{k} \pm 2 \varkappa| \pm 2 \varkappa_{0}\right)$. The appearance of these final Dirac deltas is intrinsically connected with the monochromaticity of the strong wave. The energetic balances imposed by them cannot be fulfilled in general, leading to vanishing rates. For finite pulses the situation is somewhat different (details can be found in refs. [57, 77, 78]).
} 
finds that

$$
\pi_{ \pm}\left(n_{*}, \xi_{\epsilon}\right)=\frac{\alpha_{\epsilon}}{2 \pi} m_{\epsilon}^{2} \int_{-1}^{1} d v \int_{0}^{\infty} \frac{d \rho}{\rho} \Omega_{ \pm} \exp \left\{-\frac{2 i \rho n_{*}}{\left(1+\xi_{\epsilon}^{2}\right)\left(1-v^{2}\right)}\left[1+2 A \xi_{\epsilon}^{2}\right]\right\} .
$$

While $\alpha_{\epsilon} \equiv \epsilon^{2} e^{2}=\epsilon^{2} / 137$ denotes the fine structure constant relative to the MCPs,

$$
n_{*}=2 \frac{m_{\epsilon}^{2}\left(1+\xi_{\epsilon}^{2}\right)}{k \varkappa} \quad \text { with } \quad \xi_{\epsilon}^{2}=-\frac{\epsilon^{2} e^{2} a^{2}}{m_{\epsilon}^{2}}
$$

refers to the threshold parameter for the photo-production of a $q_{\epsilon}^{+} q_{\epsilon}^{-}$-pair.

The difference between each eigenvalue [eq. (2.14)] is originally introduced by the functions $\Omega_{ \pm}$:

$$
\Omega_{ \pm}=2 \xi_{\epsilon}^{2} \frac{1+v^{2}}{1-v^{2}}\left[\sin ^{2}(\rho) \pm 2 i \rho A_{0}\right]-1+\exp (i y) .
$$

Other functions and parameters contained in the above expressions, are given by

$$
A=\frac{1}{2}\left[1-\frac{\sin ^{2}(\rho)}{\rho^{2}}\right], \quad A_{0}=\frac{1}{2}\left[\frac{\sin ^{2}(\rho)}{\rho^{2}}-\frac{\sin (2 \rho)}{2 \rho}\right], \quad y=\frac{4 n_{*} \xi_{\epsilon}^{2} \rho A}{\left(1+\xi_{\epsilon}^{2}\right)\left(1-v^{2}\right)} .
$$

With the change of variable $\left(1-v^{2}\right)^{-1} \rightarrow \frac{1}{2}[\cosh (t)+1]$ and the succeding identification of the Hankel functions of second kind $\mathrm{H}_{\nu}^{(2)}(z)=\frac{2 i}{\pi} \exp \left[\frac{i}{2} \pi \nu\right] \int_{0}^{\infty} d t \exp [-i z \cosh (t)] \cosh (\nu t)$, the variable $v$ is integrated out and the $\Pi_{0}^{\mu \nu}$-eigenvalues acquire the compact structures

$$
\pi_{ \pm}\left(n_{*}, \xi_{\epsilon}\right)=\frac{1}{2} \alpha_{\epsilon} m_{\epsilon}^{2} \int_{0}^{\infty} \frac{d \rho}{\rho} \Upsilon_{ \pm} \exp (-i \eta)
$$

where $\eta \equiv \rho n_{*}\left(1+2 \xi_{\epsilon}^{2} A\right) /\left(1+\xi_{\epsilon}^{2}\right)$ and the functions $\Upsilon_{ \pm}$read

$$
\begin{aligned}
\Upsilon_{ \pm}= & \left\{\eta\left[\mathrm{H}_{0}^{(2)}(\eta)+i \mathrm{H}_{1}^{(2)}(\eta)\right]-i \mathrm{H}_{0}^{(2)}(\eta)\right\}\left[2 \xi_{\epsilon}^{2} \sin ^{2}(\rho) \pm 4 i \xi_{\epsilon}^{2} \rho A_{0}\right]+\eta\left[\mathrm{H}_{0}^{(2)}(\eta)+i \mathrm{H}_{1}^{(2)}(\eta)\right] \\
& -\rho n_{*}\left[\mathrm{H}_{0}^{(2)}\left(\rho n_{*}\right)+i \mathrm{H}_{1}^{(2)}\left(\rho n_{*}\right)\right] \exp \left[\frac{2 i \xi_{\epsilon}^{2} \rho n_{*} A}{1+\xi_{\epsilon}^{2}}\right] .
\end{aligned}
$$

In order to pursue our analysis we seek the flavor-like solutions of the problem as superpositions of tranverse eigenwaves with opposite helicities

$$
a^{\mu}(k)=f_{+}(k) \Lambda_{+}^{\mu}+f_{-}(k) \Lambda_{-}^{\mu} \quad \text { and } \quad w^{\mu}(k)=g_{+}(k) \Lambda_{+}^{\mu}+g_{-}(k) \Lambda_{-}^{\mu} .
$$

Eqs. (2.12)-(2.20) are then inserted into eq. (2.10) and (2.11). As a consequence, the problem defined by the latter formulae splits into two eigenproblems; each one associated with a unique value of helicity as one can expect from the angular-momentum conservation

$$
\left[\begin{array}{cc}
k^{2}-\pi_{ \pm} & \frac{1}{\chi} \pi_{ \pm} \\
\frac{1}{\chi} \pi_{ \pm} & k^{2}-\frac{1}{\chi^{2}} \pi_{ \pm}
\end{array}\right]\left[\begin{array}{l}
f_{ \pm}(k) \\
g_{ \pm}(k)
\end{array}\right]=0 .
$$

Next, the leading terms in the diagonal elements are linearized according to the rule $k^{2} \simeq$ $2 \omega_{\boldsymbol{k}}\left(w-\omega_{\boldsymbol{k}}\right)$ with $\omega_{\boldsymbol{k}} \equiv|\boldsymbol{k}|$, which is equivalent to reducing the order in the differential versions of the equations of motion [eq. (2.10) and (2.11)] (see section 2.4). The dispersion 
relations are then established by setting the determinants of the resulting matrices to zero. Explicitly, we obtain

$$
w_{ \pm}^{(\gamma)}=\omega_{\boldsymbol{k}}+\frac{\pi_{ \pm}}{2 \omega_{\boldsymbol{k}}} \quad \text { and } \quad w_{ \pm}^{\left(\gamma^{\prime}\right)}=\omega_{\boldsymbol{k}}+\frac{\pi_{ \pm}}{2 \chi^{2} \omega_{\boldsymbol{k}}} .
$$

Hereafter the symbols $\gamma$ and $\gamma^{\prime}$ label the dispersion laws associated with the physical modes of visible and hidden-photon fields, respectively. Note that the contributions resulting from the off-diagonal terms have been ignored because they provide corrections smaller by a factor $\sim(\epsilon e)^{2} e_{h}^{2}$.

Owing to the non-hermiticity of the vacuum polarization tensor, its eigenvalues can be decomposed in terms of their real and imaginary parts $\pi_{ \pm}=\operatorname{Re} \pi_{ \pm}+i \operatorname{Im} \pi_{ \pm}$. This fact renders the dispersion relations [eq. (2.22)] complex functions too with $w_{ \pm}=\operatorname{Re} w_{ \pm}+$ $i \operatorname{Im} w_{ \pm}$. As a consequece, we can define the vacuum refractive indices $n_{ \pm}=|\boldsymbol{k}| / \operatorname{Re} w_{ \pm}$and the corresponding absorption coefficients $\kappa_{ \pm} \equiv-\operatorname{Im} w_{ \pm}$associated with each propagating mode of the respective Abelian fields:

$$
\begin{array}{cc}
n_{ \pm}-1 & =-\left.\frac{\operatorname{Re} \pi_{ \pm}}{2 \omega_{k}^{2}}\right|_{k^{2}=0}=\left.\frac{\operatorname{Re} \alpha_{3} \mp \operatorname{Im} \alpha_{1}}{2 \omega_{k}^{2}}\right|_{k^{2}=0}, \quad n_{ \pm}^{\left(\gamma^{\prime}\right)}-1=\frac{1}{\chi^{2}}\left(n_{ \pm}-1\right), \\
\kappa_{ \pm}=-\left.\frac{\operatorname{Im} \pi_{ \pm}}{2 \omega_{\boldsymbol{k}}}\right|_{k^{2}=0}=\left.\frac{\operatorname{Im} \alpha_{3} \pm \operatorname{Re} \alpha_{1}}{2 \omega_{\boldsymbol{k}}}\right|_{k^{2}=0}, & \kappa_{ \pm}^{\left(\gamma^{\prime}\right)}=\frac{1}{\chi^{2}} \kappa_{ \pm},
\end{array}
$$

where the decomposition $\pi_{ \pm}=-\left(\alpha_{3} \pm i \alpha_{1}\right)$ has been used. Observe that the terms proportional to the real and imaginary parts of $\alpha_{1}$ determine the degree of dichroism and birefringence of the vacuum polarized by the external laser wave [eq. (2.4)], respectively. The former phenomenon is closely associated with the different amount of pairs of MCPs produced by each propagating mode. In the field of the wave, the production thresholds are determined by the condition $n \geqslant n_{*}$ [see eq. (2.15)] with $n$ denoting the minimal number of photons from the strong wave that kinematically allows the multiphoton process $k+n \varkappa \rightarrow q_{\epsilon}^{+}+q_{\epsilon}^{-}$. Note that the previous relation leads to a condition $m_{\epsilon} \leqslant m_{n}$ which depends on the threshold mass

$$
m_{n} \equiv \sqrt{\frac{1}{2} n k \varkappa-\epsilon^{2} m^{2} \xi^{2}},
$$

with $\xi^{2}=-e^{2} a^{2} / m^{2}$ refering to the usual laser intensity parameter with $m$ being the electron mass. Clearly, eq. (2.24) provides real threshold masses whenever the condition $n \varkappa k /\left(2 m^{2} \xi^{2}\right) \geqslant \epsilon^{2}$ is satisfied. Close to the lowest thresholds of pair production of MCPs $\left[n_{*} \sim 1\right]$ the chiral birefringence and dichroism properties of the vacuum are predicted to be considerably more pronounced than in the cases asymptotically far from it $\left[n_{*} \rightarrow \infty\right.$ and $\left.n_{*} \rightarrow 0\right]$, at which the vacuum behaves like a nonabsorbing isotropic medium [57]. In the following our attention is focused on the simple cases in which one or two photons from the strong wave $[n=1,2]$ are absorbed, i. e., the limits of the two $(k+\varkappa)$ and three-photon $(k+2 \varkappa)$ reactions. Contributions of higher thresholds $[n>2]$ are beyond the scope of this work. This is partially motivated by the fact that for $\xi_{\epsilon}<1$ the photo-production rate at higher thresholds scales as $\mathcal{R}_{n} \propto \xi_{\epsilon}^{2 n}[66,80]$, which provides an evidence that the production of $q_{\epsilon}^{+} q_{\epsilon}^{-}$pairs by means of the absorption of several photons from the external wave is less likely to occur. 


\subsection{Spectral decomposition at $\xi_{\epsilon}<1$}

The integrands which define the $\Pi_{0}^{\mu \nu}$-eigenvalues are functions of the variable $\eta=n_{*} \rho(1-$ $\Delta)$ with $\Delta=\frac{\xi_{\epsilon}^{2}}{\left(1+\xi_{\epsilon}^{2}\right)} \frac{\sin ^{2}(\rho)}{\rho^{2}}$. In the region of interest $\left[\xi_{\epsilon}<1\right]$, this factor is much smaller than unity and the respective Taylor expansions of the integrands lead to a sum of contributions coming from the threshold points. To show this we first consider the lowest order terms with respect to $\Delta$. Once the integral representations of the remainig Hankel functions $\mathrm{H}_{0,1}^{(2)}\left(\rho n_{*}\right)$ are used [see below eq. (2.17)], we can exchange the order of integration and first integrate over $\rho .{ }^{4}$ The real parts of the resulting integrands turn out to be discontinous functions at $n_{*}=1$ and determine the leading order terms of the absorption coefficients. After having integrated out the remaining integration variable, they read

$$
\begin{aligned}
& \kappa_{+, 1}=\frac{\alpha_{\epsilon} m_{\epsilon}^{2} \xi_{\epsilon}^{2}}{4 \omega_{k}}\left\{\frac{1-v_{1}^{4}}{2\left(1+\xi_{\epsilon}^{2}\right)} \ln \left(\frac{1+v_{1}}{1-v_{1}}\right)+2 v_{1}\left(1-\frac{1-v_{1}^{2}}{2\left(1+\xi_{\epsilon}^{2}\right)}\right)\right\} \Theta\left[v_{1}^{2}\right], \\
& \kappa_{-, 1}=\frac{\alpha_{\epsilon} m_{\epsilon}^{2} \xi_{\epsilon}^{2}}{4 \omega_{k}}\left\{\left(2+\frac{1-v_{1}^{4}}{2\left(1+\xi_{\epsilon}^{2}\right)}\right) \ln \left(\frac{1+v_{1}}{1-v_{1}}\right)-4 v_{1}\left(1+\frac{1-v_{1}^{2}}{4\left(1+\xi_{\epsilon}^{2}\right)}\right)\right\} \Theta\left[v_{1}^{2}\right] .
\end{aligned}
$$

Here $\Theta[x]$ represents the unit step function, whereas $v_{1}=\left(1-n_{*}\right)^{1 / 2}$ is closely connected to the relative speed of the final particle states when only one photon of the intense laser wave has been absorbed $;^{5}$ hence the use of the lower index 1 . We emphasize that eqs. (2.25)(2.26) provide nonvanishing contributions whenever the MCP mass $m_{\epsilon}$ is smaller or equal to the first threshold mass $m_{1}=\left(k \varkappa / 2-\epsilon^{2} m^{2} \xi^{2}\right)^{1 / 2}$, corresponding to $n_{*} \leqslant 1$.

In contrast to the previous case, the imaginary parts of the $\pi_{ \pm}$-integrands are continuous functions which define the leading terms of the vacuum refractive indices. The explicit expressions of these optical entities are difficult to obtain. Asymptotic expressions for $n_{*} \ll 1$ and $n_{*} \approx 1$ can be found but both cases have restricted validity in comparison to eqs. (2.25)-(2.26). Because of this reason, we have opted to express them as parametric integrals:

$$
\begin{aligned}
n_{ \pm}-1 \simeq & \pm \frac{\alpha_{\epsilon} m_{\epsilon}^{2} \xi_{\epsilon}^{2}}{2 \pi \omega_{\boldsymbol{k}}^{2}} \int_{0}^{1} d v\left\{\left[1-\frac{2 \varrho}{n_{*}}\left(1 \mp \frac{n_{*}}{1+\xi_{\epsilon}^{2}}\right)\right] \ln \left(\frac{1+\varrho}{|1-\varrho|}\right)^{1 / 2}\right. \\
& \left.\mp\left[1-\frac{2 \varrho}{n_{*}}\left(1 \mp n_{*}\right)+\frac{2 \varrho^{2}}{1+\xi_{\epsilon}^{2}}\left(1 \mp \frac{2\left(1+\xi_{\epsilon}^{2}\right)}{n_{*}}\right)\right] \ln \left(\frac{|\varrho|}{\sqrt{\left|1-\varrho^{2}\right|}}\right)\right\},
\end{aligned}
$$

where $\varrho \equiv \varrho\left(v, n_{*}\right)=n_{*}\left(1-v^{2}\right)^{-1}$ is a function of both the integration variable $v$ and the threshold parameter $n_{*}$.

Equation (2.27) deserves further analysis. To simplify it, we only keep the quadratic proportionality on $\xi_{\epsilon}$ in $n_{ \pm}-1$ focusing on the Born approximation $\left[\xi_{\epsilon}^{2} \ll 1\right]$. As a consequence, the behavior of the integrand of $n_{ \pm}$as $v \rightarrow 0\left[\varrho \rightarrow n_{*}\right]$ turns out to be

$$
\stackrel{v \rightarrow 0}{\longrightarrow}-\left[1 \mp 2 n_{*}\right] \ln \left(\frac{1+n_{*}}{1-n_{*}}\right)^{1 / 2} \pm\left[1 \pm 2 n_{*}-2 n_{*}^{2}\right] \ln \left(\frac{n_{*}}{\sqrt{1-n_{*}^{2}}}\right) .
$$

\footnotetext{
${ }^{4}$ The procedure outlined here shares several similarities with the method applied by the authors in [57], particularly in those issues associated with the integrations over the variable $\rho$. The reader interested in the details of such operations may find it helpful to refer to the aforementioned reference.

${ }^{5}$ In the center-of-mass frame, when $n$ photons from the laser field are absorbed, the relative speed between the final particles is given by $\left|\boldsymbol{v}_{\text {rel }}\right|=\left|\boldsymbol{v}_{q_{\epsilon}^{-}}-\boldsymbol{v}_{q_{\epsilon}^{+}}\right|=2 v_{n}$ with $v_{n}=\left(1-n_{*} / n\right)^{1 / 2}$.
} 
Conversely, when $v \rightarrow 1$ the function $\varrho \rightarrow \infty$ and the integrand associated with $n_{ \pm}$tends to \pm 1 . The situation is different at $v \rightarrow \sqrt{1-n_{*}}<1$ with $n_{*} \in(0,1)$, i. e., $\varrho \rightarrow 1$, since the derivation of $\pi_{ \pm}$relies on integrals tabulated in ref. [81], which apply whenever $\varrho \neq 1$, for further details see [57]. Therefore, this singularity is actually not reached and, in a neighborhood of this point the integrand of $n_{ \pm}$behaves as $\sim \frac{1}{2}\left(1 \pm 1 \mp \frac{2}{n_{*}}\right) \ln |1-\varrho|$. Note that, due to the exclusion of the aforementioned point, the integral over $v$ in eq. (2.27) must be understood as a Cauchy principal value. However, if $n_{*} \gtrsim 1$ the square root of $1-n_{*}$ is an imaginary quantity. As a consequence, there is no singularity within the integration region $0 \leqslant v \leqslant 1$ and - in contrast to eqs. (2.25)-(2.26) - the leading order terms of the vacuum refractive indices turn out to be dominant, even when the contributions resulting from higher thresholds are taken into account.

Of particular interest for us are the corrections to eqs. (2.25)-(2.26) which result from the absorption of two photons from the strong wave. To determine them we assume that $1<n_{*} \leq 2$, so that the $\xi_{\epsilon}^{4}$-correction due to two-photon reaction $(k+\varkappa)$ is excluded. With this detail in mind we go one step further in the $\Delta$-expansion of eqs. (2.18)-(2.19). We then find that the real parts of the integrands associated with the three-photon reaction $(k+2 \varkappa)$ are, in general, discontinuous functions at the point where $n_{*}=2$. Following the procedure described above we obtain

$$
\kappa_{ \pm, 2}=\frac{\alpha_{\epsilon} m_{\epsilon}^{2} \xi_{\epsilon}^{4}}{4 \omega_{\boldsymbol{k}}\left(1+\xi_{\epsilon}^{2}\right)}\left[\mathcal{F}_{1}\left(\tau_{2}\right)+2 \frac{1-v_{2}^{2}}{1+\xi_{\epsilon}^{2}} \mathcal{F}_{2}\left(v_{2}\right) \pm \mathcal{F}_{3}\left(v_{2}\right)\right] \Theta\left[v_{2}^{2}\right]
$$

where $v_{2}=\left(1-n_{*} / 2\right)^{1 / 2}$ defines the relative speed between the produced minicharges [see footnote 4], whereas the functions $\mathcal{F}_{i}\left(v_{2}\right)$ with $i=1,2,3$ are given by

$$
\begin{aligned}
& \mathcal{F}_{1}\left(v_{2}\right)=v_{2}\left(1+v_{2}^{2}\right)-\left(1-v_{2}^{2}\right)^{2} \operatorname{arctanh}\left(v_{2}\right) \\
& \mathcal{F}_{2}\left(\tau_{2}\right)=\frac{1}{12} v_{2}\left(15 v_{2}^{4}-4 v_{2}^{2}-3\right)+\frac{1}{4}\left(1+v_{2}^{2}+3 v_{2}^{4}-5 v_{2}^{6}\right) \operatorname{arctanh}\left(v_{2}\right) \\
& \mathcal{F}_{3}\left(\tau_{2}\right)=-\frac{1}{3} v_{2}\left(6 v_{2}^{4}-7 v_{2}^{2}+3\right)+\left(1-3 v_{2}^{4}+2 v_{2}^{6}\right) \operatorname{arctanh}\left(v_{2}\right)
\end{aligned}
$$

The above formulae allow us to determine the asymptotic expression of $\kappa_{ \pm, 2}$ as $n_{*} \rightarrow 2^{-}$, i. e., when the particles are created in the center-of-mass frame almost at rest $\left[v_{2} \sim 0\right]$. In this limit the functions $\mathcal{F}_{i}\left(v_{2}\right)$ are dominated by cubic dependences on $\tau_{2}$ and the absorption coefficients approach to $\kappa_{ \pm, 2} \approx \alpha_{\epsilon} m_{\epsilon}^{2} \xi_{\epsilon}^{4} v_{2}^{3}(8 \mp 1) /\left[12 \omega_{k}\left(1+\xi_{\epsilon}^{2}\right)\right]$. Conversely, when $n_{*} \rightarrow 1^{+}$, i. e., $\left[v_{2} \rightarrow 1 / \sqrt{2}\right]$, we find the asymptotes $\kappa_{ \pm, 2} \approx \alpha_{\epsilon} m_{\epsilon}^{2} \xi_{\epsilon}^{4}(0.4 \mp 0.1) /\left[4 \omega_{\boldsymbol{k}}\right]$, provided the condition $\xi_{\epsilon} \ll 1$ holds. The asymptotic behavior of $\kappa_{ \pm, 1}$ was derived previously and can be found in ref. [57]

Finally, we recall that the imaginary part of the polarization tensor is associated with the production rate of a $q_{\epsilon}^{+} q_{\epsilon}^{-}$pair through the optical theorem. Within the accuracy to the second order with respect to the radiative corrections, the total creation rate $\mathcal{R}$ of a $q_{\epsilon}^{+} q_{\epsilon}^{-}$pair from a photon - averaged over the polarization states $\Lambda_{ \pm}^{\mu}$ - is fully determined by the absorption coefficients [57]. Explicit polarization operator approaches to the rate associated with the two-photon reaction may be found in separate papers (see refs. [79] and [82]). In the limit of $\xi_{\epsilon} \ll 1$ it was obtained that $\mathcal{R}_{1} \propto \xi_{\epsilon}^{2}$. When inspecting eq. (2.29) 
one can easily establish that the average rate for producing a $q_{\epsilon}^{+} q_{\epsilon}^{-}$pair in a three-photon reaction is $\mathcal{R}_{2} \propto \xi_{\epsilon}^{4}$, a fact which verifies the last comment in section 2.2 .

\subsection{Conversion probability and polarimetric observables}

The linearization used in the derivation of eq. (2.22) is also a convenient simplification for solving the initial system of differential equations [eqs. (2.10) and (2.11)]. It turns out to be appropriate to seek for solutions in the form of plane waves $\sim e^{i \boldsymbol{k} \cdot \boldsymbol{x}-i \omega_{\boldsymbol{k}} t}$. This fact allows us to approximate the Laplacian involved in the equations of motion [see below eq. (2.8)] by a first order differential operator according to the rule $\partial^{2} / \partial t^{2}+\boldsymbol{k}^{2}=$ $(i \partial / \partial t+|\boldsymbol{k}|)(-i \partial / \partial t+|\boldsymbol{k}|) \simeq 2 \omega_{\boldsymbol{k}}\left(-i \partial / \partial t+\omega_{\boldsymbol{k}}\right)$. As a consequece, the boundary conditions on the derivatives of both gauge fields can be ignored and the problem under consideration reduces to solve the equation

$$
-i \frac{\partial}{\partial t}\left[\begin{array}{c}
f_{ \pm}(\boldsymbol{k}, t) \\
g_{ \pm}(\boldsymbol{k}, t)
\end{array}\right]=\left[\begin{array}{cc}
w_{ \pm}^{(\gamma)} & \frac{1}{2 \chi \omega_{\boldsymbol{k}}} \pi_{ \pm} \\
\frac{1}{2 \chi \omega_{\boldsymbol{k}}} \pi_{ \pm} & w_{ \pm}^{\left(\gamma^{\prime}\right)}
\end{array}\right]\left[\begin{array}{l}
f_{ \pm}(\boldsymbol{k}, t) \\
g_{ \pm}(\boldsymbol{k}, t)
\end{array}\right]
$$

We stress that the diagonal elements of the matrix in eq. (2.33) are the dispersion relations given in eq. (2.22).

The solution of the above equation can be written as a superposition of eigenvectors of the linearized version of eq. (2.21). In fact, by introducing the mixing angle $\varphi \equiv$ $\arctan \left(\frac{\chi}{1-\chi^{2}}\right)$ we find

$$
\left[\begin{array}{l}
f_{ \pm}(k, t) \\
g_{ \pm}(k, t)
\end{array}\right]=\frac{\mathcal{C}_{ \pm}^{(\gamma)}}{\sqrt{1+\tan ^{2}(\varphi)}}\left[\begin{array}{c}
1 \\
\tan (\varphi)
\end{array}\right] e^{-i w_{ \pm}^{(\gamma)} t}-\frac{\mathcal{C}_{ \pm}^{\left(\gamma^{\prime}\right)}}{\sqrt{1+\tan ^{2}(\varphi)}}\left[\begin{array}{c}
\tan (\varphi) \\
-1
\end{array}\right] e^{-i w_{ \pm}^{\left(\gamma^{\prime}\right)} t}
$$

The constants $\mathcal{C}_{ \pm}^{\left(\gamma, \gamma^{\prime}\right)}$ are determined by supposing an experimental setup which starts at $t=0$ - without a hidden-photon field but with an incoming electromagnetic probe beam of finite amplitude $f_{ \pm}(\boldsymbol{k}, 0)=\left[4 \pi /\left(2 \omega_{\boldsymbol{k}}\right)\right]^{1 / 2}$. With this idea in mind, we obtain a system of algebraic equations for $\mathcal{C}_{ \pm}^{\left(\gamma, \gamma^{\prime}\right)}$, whose solutions allow us to express the flavor-like components as

$$
f_{ \pm}(\boldsymbol{k}, t)=\sqrt{\frac{4 \pi}{2 \omega_{\boldsymbol{k}}}} \mathcal{A}_{ \pm}(\boldsymbol{k}, t) e^{-i \omega_{\boldsymbol{k}} t} \quad \text { and } \quad g_{ \pm}(\boldsymbol{k}, t)=\sqrt{\frac{4 \pi}{2 \omega_{\boldsymbol{k}}}} \mathcal{B}_{ \pm}(\boldsymbol{k}, t) e^{-i \omega_{\boldsymbol{k}} t}
$$

where - in the limit of weak-mixing $\chi \ll 1$ and $\varphi \simeq \chi$ - the wave amplitudes $\mathcal{A}_{ \pm}(\boldsymbol{k}, t)$ and $\mathcal{B}_{ \pm}(\boldsymbol{k}, t)$ approach to the following expressions

$$
\begin{aligned}
\mathcal{A}_{ \pm}(\boldsymbol{k}, t) \approx & \exp \left\{i\left(n_{ \pm}-1\right) \omega_{\boldsymbol{k}} t+i \chi^{2} \sin \left(\frac{n_{ \pm}-1}{\chi^{2}} \omega_{\boldsymbol{k}} t\right) \exp \left(-\frac{1}{\chi^{2}} \kappa_{ \pm} t\right)\right. \\
& \left.-\kappa_{ \pm} t-\chi^{2}\left[1-\cos \left(\frac{n_{ \pm}-1}{\chi^{2}} \omega_{\boldsymbol{k}} t\right) \exp \left(-\frac{1}{\chi^{2}} \kappa_{ \pm} t\right)\right]\right\}, \\
\mathcal{B}_{ \pm}(\boldsymbol{k}, t) \approx & \chi\left\{\exp \left(-\frac{1}{\chi} \kappa_{ \pm} t\right)-\cos \left(\frac{n_{ \pm}-1}{\chi^{2}} \omega_{\boldsymbol{k}} t\right)+i \sin \left(\frac{n_{ \pm}-1}{\chi^{2}} \omega_{\boldsymbol{k}} t\right)\right\},
\end{aligned}
$$

with $n_{ \pm}$and $\kappa_{ \pm}$given in eq. (2.23). 
The modulus square of $\mathcal{B}_{ \pm}(\boldsymbol{k}, t)$ gives us the conversion probability, which turns out to be intrinsically associated with the exponentials responsible for the damping of the corresponding electromagnetic waves due to the photon-paraphoton oscillations

$$
\mathcal{P}_{\gamma_{ \pm} \rightarrow \gamma_{ \pm}^{\prime}}(\tau) \simeq \chi^{2}\left\{1+\exp \left(-\frac{2}{\chi^{2}} \kappa_{ \pm} \tau\right)-2 \exp \left(-\frac{1}{\chi^{2}} \kappa_{ \pm} \tau\right) \cos \left(\frac{n_{ \pm}-1}{\chi^{2}} \omega_{\boldsymbol{k}} \tau\right)\right\} .
$$

Observe that this formula has been evaluated at the pulse length $t=\tau$ of the external laser wave [eq. (2.4)]. Interestingly, it resembles the probability of conversion resulting from a setup in which a constant magnetic field drives the photon-paraphoton oscillations $[11,12]$. Note that eq. $(2.38)$ is characterized by an oscillatory pattern which tends to be exponentially suppressed as the pulse length $\tau$ of the laser wave is much larger than the characteristic time of the transition process $\sim \chi^{2} \kappa_{ \pm}^{-1}$. In such a case, eq. (2.38) asymptotically approaches $\mathcal{P}_{\gamma_{ \pm} \rightarrow \gamma_{ \pm}^{\prime}}(\tau) \simeq \chi^{2}$. Conversely, when the attenuation factors $\sim \kappa_{ \pm} \tau / \chi^{2}$ and the trigonometric argument $\left(n_{ \pm}-1\right) \omega_{\boldsymbol{k}} \tau / \chi^{2}$ are much smaller than unity, the probability of conversion reduces to

$$
\mathcal{P}_{\gamma_{ \pm} \rightarrow \gamma_{ \pm}^{\prime}} \approx \frac{1}{\chi^{2}}\left[\left(n_{ \pm}-1\right)^{2} \omega_{k}^{2}+\kappa_{ \pm}^{2}\right] \tau^{2}
$$

It is worth mentioning that this expression coincides with the outcome resulting from perturbation theory when the Abelian fields in eq. (2.6) are canonically quantized. In this context, the probability amplitude of the photon-paraphoton oscillation can be read off directly from the off-diagonal elements in eq. (2.7). Within the accuracy to the second order with respect to the radiative corrections, it is explicitly given by

$$
T_{e^{(i)} k^{\prime}, e^{(f)} k}=\frac{i}{\chi} \frac{e_{\mu}^{(f)} \Pi^{\mu \nu}\left(k, k^{\prime}\right) e_{\nu}^{(i)}}{2 V\left(\omega_{\boldsymbol{k}^{\prime}} \omega_{\boldsymbol{k}}\right)^{1 / 2}} .
$$

Here $V$ denotes the normalization volume, whereas $e_{\mu}^{(i)}$ and $e_{\mu}^{(f)}$ are the initial and final polarization states, respectively. We suppose the former to be associated with visible photons [eq. (2.20)] so that $e^{(i)}=\Lambda_{ \pm}$. In contrast, the respective polarizations of the final hidden $\mathrm{U}(1)$-gauge states are chosen as $e^{(f)}=\Lambda_{ \pm}^{*}$. We insert these and the expression for the polarization tensor [eq. (2.12)] into the eq. (2.40). Consequently, the probability amplitude becomes

$$
T_{\gamma_{ \pm} \rightarrow \gamma_{ \pm}^{\prime}}=-\frac{i}{\chi} \frac{\pi_{ \pm}}{2 V \omega_{k}}(2 \pi)^{4} \delta^{4}\left(k^{\prime}-k\right) .
$$

Next, the modulus squared of this formula is integrated over the momentum of the final hidden-photon field. Such a procedures allows us to write the conversion probability as in eq. (2.39), provided the usual interpretation of the interacting time $\tau \equiv 2 \pi \delta(0)$ is used, where the occurence of a vanishing argument in the Dirac delta is a direct consequence of the energy conservation in the process.

We want to conclude this section by deriving the potential observables in an optical experiment assisted by the field of a circularly polarized plane wave. To this end we emphasize that, in this kind of background, the vacuum behaves as a chiral medium rather than an uniaxial material. In correspondence, the polarization plane of an incoming linearly 
polarized probe beam undergoes a rotation $\vartheta(\tau)$ due to the relative phase difference between the propagating (helicity) modes [eq. (2.35)]:

$$
\begin{aligned}
|\vartheta(\tau)| \approx & \frac{1}{2} \mid\left(n_{+}-n_{-}\right) \omega_{\boldsymbol{k}} \tau+\chi^{2} \sin \left(\frac{n_{+}-1}{\chi^{2}} \omega_{\boldsymbol{k}} \tau\right) \exp \left(-\frac{1}{\chi^{2}} \kappa_{+} \tau\right) \\
& -\chi^{2} \sin \left(\frac{n_{-}-1}{\chi^{2}} \omega_{\boldsymbol{k}} \tau\right) \exp \left(-\frac{1}{\chi^{2}} \kappa_{-} \tau\right) \mid \ll 1 .
\end{aligned}
$$

As such, the interaction with the strong field of the wave transforms the outgoing probe beam into an elliptically polarized wave. In our context, the degree of ellipticity $\psi(\tau)$ is an outcome of both the absorption of visible waves via the production of pairs of MCPs and their conversion into hidden-photons. Hence, it is determined by the difference between the attenuation coefficients of the visible circular modes [eq. (2.35)]. Explicitly,

$$
\begin{aligned}
|\psi(\tau)| \approx & \frac{1}{2} \mid\left(\kappa_{-}-\kappa_{+}\right) \tau+\chi^{2} \cos \left(\frac{n_{+}-1}{\chi^{2}} \omega_{\boldsymbol{k}} \tau\right) \exp \left(-\frac{1}{\chi^{2}} \kappa_{+} \tau\right) \\
& -\chi^{2} \cos \left(\frac{n_{-}-1}{\chi^{2}} \omega_{\boldsymbol{k}} \tau\right) \exp \left(-\frac{1}{\chi^{2}} \kappa_{-} \tau\right) \mid \ll 1 .
\end{aligned}
$$

As in the case of the conversion probability, the oscillatory pattern in both observables is suppressed when very long pulses $\chi^{2} \kappa_{ \pm}^{-1} \ll \tau$ are considered. The resulting asymptotes coincide with the standard results for the ellipticity and rotation in a pure MCPs model [57]. However, the effects resulting from the photon-paraphoton oscillations could be quite noticeable if the pulse length is much smaller than $\chi^{2} \kappa_{ \pm}^{-1}$ and if it simultaneously satisfies the condition $\tau \ll \chi^{2} \omega_{\boldsymbol{k}}^{-1}\left(n_{ \pm}-1\right)^{-1}$. Then eqs. (2.42) and (2.43) approach

$$
\begin{aligned}
|\vartheta(\tau)| & \approx\left|\left(n_{+}-n_{-}\right) \omega_{\boldsymbol{k}} \tau+\frac{1}{4 \chi^{2}}\left[\left(n_{-}-1\right) \kappa_{-}-\left(n_{+}-1\right) \kappa_{+}\right] \omega_{\boldsymbol{k}} \tau^{2}\right| \\
|\psi(\tau)| & \approx\left|\left(\kappa_{-}-\kappa_{+}\right) \tau+\frac{1}{4 \chi^{2}}\left[\left(n_{-}-1\right)^{2}-\left(n_{+}-1\right)^{2}\right] \omega_{\boldsymbol{k}}^{2} \tau^{2}+\frac{1}{4 \chi^{2}}\left(\kappa_{+}^{2}-\kappa_{-}^{2}\right) \tau^{2}\right| .
\end{aligned}
$$

Interestingly, the leading terms are increased by a factor two as compared to those associated with the pure MCPs model. Such a feature provides an evidence that, in the limit under consideration, the production of pairs of MCPs and the vacuum birefringence are stimulated by the existence of a hidden-photon field.

\section{$3 \quad$ Experimental prospects}

\subsection{Estimating the exclusion limits}

Restrictions on the $\left(\epsilon, m_{\epsilon}\right)$ plane can be established whenever in certain confidence levels $\psi_{\mathrm{CL} \%}, \vartheta_{\mathrm{CL} \%}$, neither rotation of the polarization plane [eqs. (2.42)] nor ellipticity of the outgoing probe beam [eq. (2.43)] are detected. We note that, while experimental data for the proposed setup do not exist yet, ellipticities and rotation angles can nowadays be measured with an accuracy of about $\sim 10^{-10} \mathrm{rad}$ in the optical regime [84]. ${ }^{6}$ Hereafter, we

\footnotetext{
${ }^{6}$ An experiment to measure vacuum birefringence by probing a Petawatt optical laser $[\xi \gg 1]$ with a x-ray free electron laser, has been proposed by the HIBEF consortium [85].
} 
give first estimates of the exclusion bounds resulting from the absence of the aforementioned signals by taking the previous value as reference for the sensitivity parameters $\psi_{\mathrm{CL} \%}, \vartheta_{\mathrm{CL} \%}$. Accordingly, we have to solve the inequalities $\psi_{\mathrm{CL} \%}>\psi(\tau)$ and $\vartheta_{\mathrm{CL} \%}>\vartheta(\tau)$ but, due to the very complicated dependence of $\psi(\tau)$ and $\vartheta(\tau)$ on the unknown parameters of our theory $\epsilon, m_{\epsilon}$ and $\chi$, no analytic solutions can be derived. Therefore, we rather determine their bounds numerically. However, in doing so we should keep in mind that the application of the expressions obtained so far requires an external laser field which approaches to our monochromatic plane-wave model [eq. (2.4)]. In practice, the monochromaticity of the high-intensity laser wave can be implemented as long as the laser-source emits a pulse with an oscillation period $\mathcal{T}=2 \pi \varkappa_{0}^{-1}$ much smaller than its temporal length $\tau$, i. e., $\varkappa_{0} \tau \gg 1$. Regarding the plane-wave character, it formally implies that the long-laser wave is infinitely extended in the plane perpendicular to the propagation direction. However, in an actual experimental realization, this condition can be considered as satisfied when the waist size of the laser beam $w_{0}$ is much greater than its wavelength $\left[w_{0} \gg \lambda_{0}\right.$ with $\left.\lambda_{0}=2 \pi \varkappa_{0}^{-1}\right]$.

In order to satisfy both conditions, we choose, for our external laser field, the set of parameters associated with the Petawatt High-Energy Laser for heavy Ion eXperiments (PHELIX) [70], currently under operation at GSI in Darmstadt, Germany. We are particularly interested in the nanosecond frontend of PHELIX $\left[w_{0} \approx 100-150 \mu \mathrm{m}\right]$, since it operates with an infrared wavelength $\lambda_{0} \simeq 1053 \mathrm{~nm}\left[\varkappa_{0} \simeq 1.17 \mathrm{eV}\right]$ and can reach a peak intensity $I \simeq 10^{16} \mathrm{~W} / \mathrm{cm}^{2}$, corresponding to a parameter $\xi \simeq 6.4 \times 10^{-2}$ in a pulse length $\tau \simeq 20$ ns. In addition, we will study the results coming from the specification of the long high-energy pulse of $400 \mathrm{~J}$ at the Laboratoire pour l'Utilisation des Lasers Intenses (LULI) [71] - currently in operation at Palaiseau, France. Similarly to the previous external source, we will focus ourselves on the nanosecond facility at $\operatorname{LULI}(2000)\left[w_{0} \sim 100 \mu \mathrm{m}\right]$, which can operate with the same central frequency as PHELIX once its fundamental harmonic is used. However, its pulse length is shorter $\tau \simeq 1.5-4$ ns and its maximum intensity is lower $I \simeq 6 \times 10^{14} \mathrm{~W} / \mathrm{cm}^{2}\left[\xi \simeq 2 \times 10^{-2}\right]$. Regarding the probe beam, we suppose that it is an optical laser obtained by coupling out a tiny fraction of the external laser wave whose intensity turns out to be much weaker than the intensity of the strong beam. We assume, in particular, that the probe frequency can be doubled $\left[\omega_{k}=2 \varkappa_{0}=2.34 \mathrm{eV}\right]$ afterwards.

Observe that, with the above assumptions, the photo-production of an electron-positron pair cannot take place. In the fields under consideration, the occurrence of a linear BreitWheeler reaction would require probe photons with energy greater than the threshold value $\omega_{k} \gtrsim m^{2} \varkappa_{0}^{-1} \approx 10 \mathrm{GeV}$. As a consequence, no contribution other than the one induced by the decay of the probe beam into MCPs pairs and its oscillation into a paraphoton is expected in the dichroic effect [eq. (2.43)]. Therefore, an eventual detection of ellipticity in the outgoing probe beam can be understood as a clear manifestation of physics beyond SM. Furthermore, below the first pair production threshold $\left[k \varkappa \ll 2 m^{2}\right]$ and for $\xi<1$, the birefringence of the pure QED vacuum is predicted to be extremely weak [57, 76]. Indeed, the rotation of the polarization plane for spinor QED is given by

$$
\left|\vartheta_{\mathrm{QED}}\right| \approx \frac{2}{315} \frac{\alpha}{\pi} \frac{(\varkappa k)^{3}}{m^{4} \omega_{k}} \xi^{2} \tau
$$



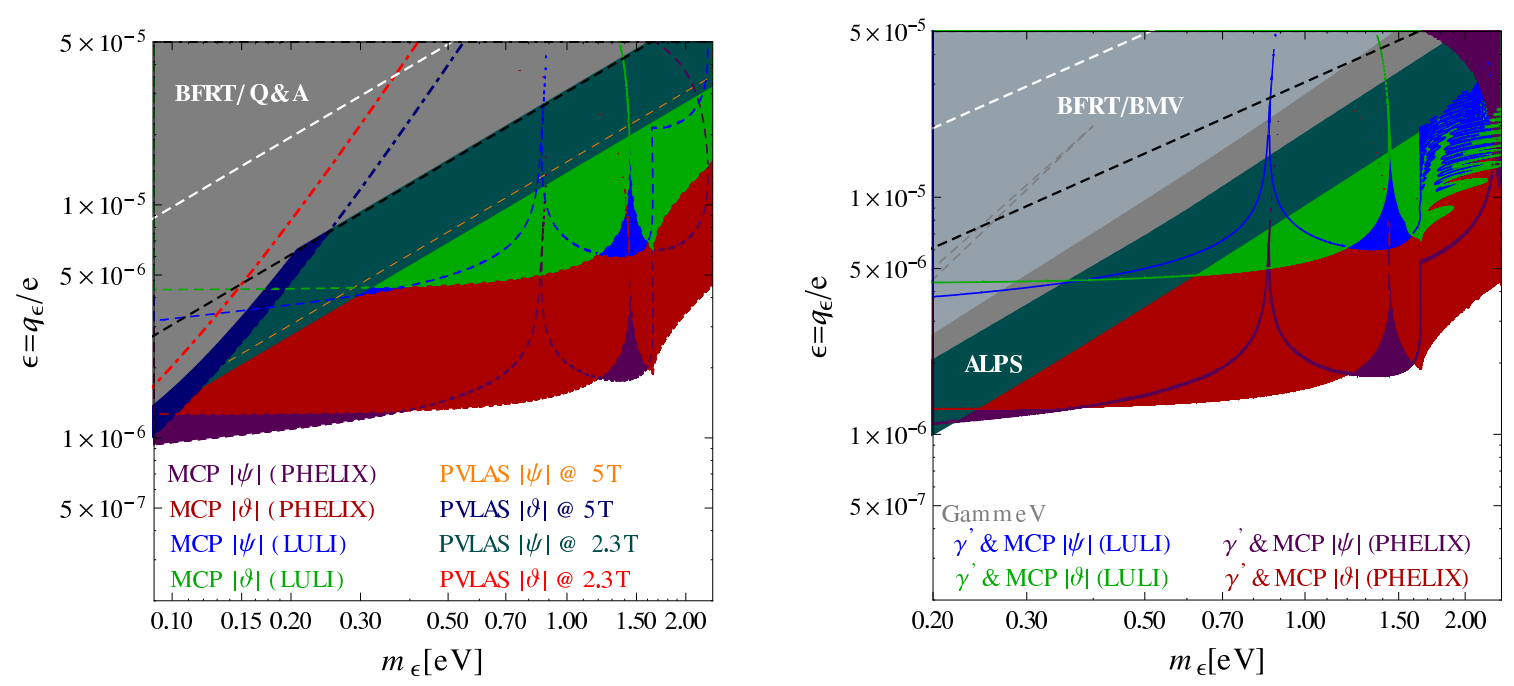

Figure 2. Projected sensitivities for MCPs of mass $m_{\epsilon}$ and relative coupling constant $\epsilon$ derived from the absence of signals in a plausible polarimetric setup assisted by a circularly polarized laser field of moderate intensity. While the left panel provides the results associated with MCPs, the right one shows the outcomes of the model including a hidden-photon field $\left(\gamma^{\prime}\right)$. In both panels the white (LULI) and black (PHELIX) dashed lines correspond to the expression $\xi_{\epsilon}=\epsilon m \xi / m_{\epsilon}=1$. The picture in the left, includes the exclusion regions coming from various experimental collaborations searching for rotation and ellipticity in a constant magnetic field such as BFRT [29], PVLAS [30, 31] and Q \& A [33]. However, the shaded areas in the upper left corner in the right panel result from different experimental collaborations dealing with the Light Shining Through a Wall mechanism. The respective $95 \%$ confidence levels needed to reproduce these results are summarized in refs. [12, 34].

When evaluating this expression with the parameters of the nanosecond frontend of PHELIX in a counter propagating geometry $[k \|-\varkappa]$, we find that its contribution $\left|\vartheta_{\text {QED }}\right| \sim$ $10^{-21}$ rad turns out to be extremely tiny in comparison with the reference sensitivity $\sim 10^{-10} \mathrm{rad}$ to be used henceforth. That the pure QED effect is so tiny is important for practical purposes, because it would allow for isolating polarization-dependent effects stemming only from the self-interaction of the electromagnetic field in vacuum mediated by hypothetical degrees of freedom. Therefore, also by sensing a rotation in the polarization plane [eq. (2.42)] other than the predictions coming from QED, we can probe the existences of our dark matter candidates.

The first estimates of the described settings are shown in figure 2 for the particular situation in which the collision is head-on, i. e., $\boldsymbol{k} \cdot \varkappa=-\omega_{\boldsymbol{k}} \varkappa_{0}$. Our exclusion regions are shaded in purple and red for PHELIX and in blue and green for LULI. We emphasize that they are expected to be trustworthy when the bounds are below the respective dashed line - white for LULI and black for PHELIX - corresponding to $\xi_{\epsilon}=\epsilon m \xi / m_{\epsilon}=1$. While the left panel displays the results coming from the pure MCP model, the discovery potential including the paraphoton effects is shown in the right panel. Note that, in the latter, the shaded regions were derived by considering the relation $\chi=\epsilon$, so that a direct comparison with the pure MCP model can be established. In contrast to the left panel, the one in the right does not show the sensitivity limits coming from the experimental 
searches of polarimetric signals. Instead, we have incorporated the upper bound obtained from the ALPS collaboration [34] which - at the time of writing - turns out to be the most stringent laboratory-based limit for MCPs in a model with massless paraphotons. Also, included are the exclusion regions resulting from other experimental collaborations dealing with the Light Shining Through a Wall mechanism such as BMV [41], BFRT [29] and GammeV [36]. Observe that in the vicinity of the first threshold mass $m_{1} \approx 1.64 \mathrm{eV},{ }^{7}$ the upper limits resulting from the search of the rotation angle are more restrictive than those arising from the ellipticity. Nearby, the bounds are $\epsilon<1.9 \times 10^{-6}$ for PHELIX and $\epsilon<6.5 \times 10^{-6}$ for LULI. Also, figure 2 verifies the statement given below eq. (2.24) about the contribution of higher thresholds, since the picture covers a region including the second threshold mass $m_{2} \approx 2.34 \mathrm{eV}$, at which the upper limit undergoes a relaxation.

In the right panel of this figure, we see that the projected sensitivities coming from the plausible absence of signals in our LULI setup follow a path very similar to the one obtained from the pure MCPs model for masses below the first threshold mass $m_{1} \approx 1.64 \mathrm{eV}$. This fact manifests a dominance of the first contributions to the observables [eqs. (2.42) and (2.43)] due to a plausible exponential suppressions of the paraphoton terms. Hence, we deduce that in such a region, the characteristic times involved in the respective damping factors of the waves $\chi^{2} \kappa_{ \pm, 1}^{-1}$ turn out to be much smaller than the laser pulse lengths $\tau \gg \chi^{2} \kappa_{ \pm, 1}^{-1}$. However, for masses embedded in the range $m_{1}<m_{\epsilon}<m_{2}$, the upper bounds resulting from LULI's parameters are characterized by fluctuating patterns which are absent in a pure MCPs scenario. The occurence of these trends is closely associated with the photon-paraphoton oscillations. In contrast to masses below $m_{1}$, the region in which $m_{1}<m_{\epsilon}<m_{2}$ turns out to feature characteristic times $\chi^{2} \kappa_{ \pm}^{-1}$ much larger than (in the case of LULI) - or at least of the order of (in the case of PHELIX) - the pulse lengths $\tau$ used. This is caused by the contributions coming from the second threshold [eq. (2.29)] which become - in the region under consideration - the leading order term in the absorption coefficients.

Actually, the dependence of the hidden gauge coupling $e_{h}$ introduces a certain level of uncertainty. Figure 3 shows how the sensitivity limits for PHELIX might vary as $e_{h}$ changes by an order of magnitude around the natural value $e_{h}=e .^{8}$ Observe that, these variations are almost imperceptible for masses below the first threshold mass. However, slight deviations in the exclusion bounds are displayed in a vicinity of the first threshold and within the interval where the photon-hidden-photon oscillations are more pronounced. Clearly, these first estimates indicate that experiments driven by long laser pulses of moderate intensities might be sensitive in regions of the parameter space which are not excluded by the outcomes of current laboratory-based collaborations such as PVLAS and BFRT. Particularly, in a vicinity of the first threshold mass, the present upper bound might be an order of magnitude more stringent than the one resulting from the PVLAS and ALPS analyses.

\footnotetext{
${ }^{7}$ Note that for the laser parameters used here, the second contribution in the threshold mass [eq. (2.24)], i.e. $\epsilon^{2} m^{2} \xi^{2} \sim 10^{-3} \mathrm{eV}^{2}$ is neglectable in comparison with the first term $k \varkappa / 2$.

${ }^{8}$ In this section and the remaining part of the paper, it must be understood that the symbol e refers to the absolute value of the electron charge.
} 


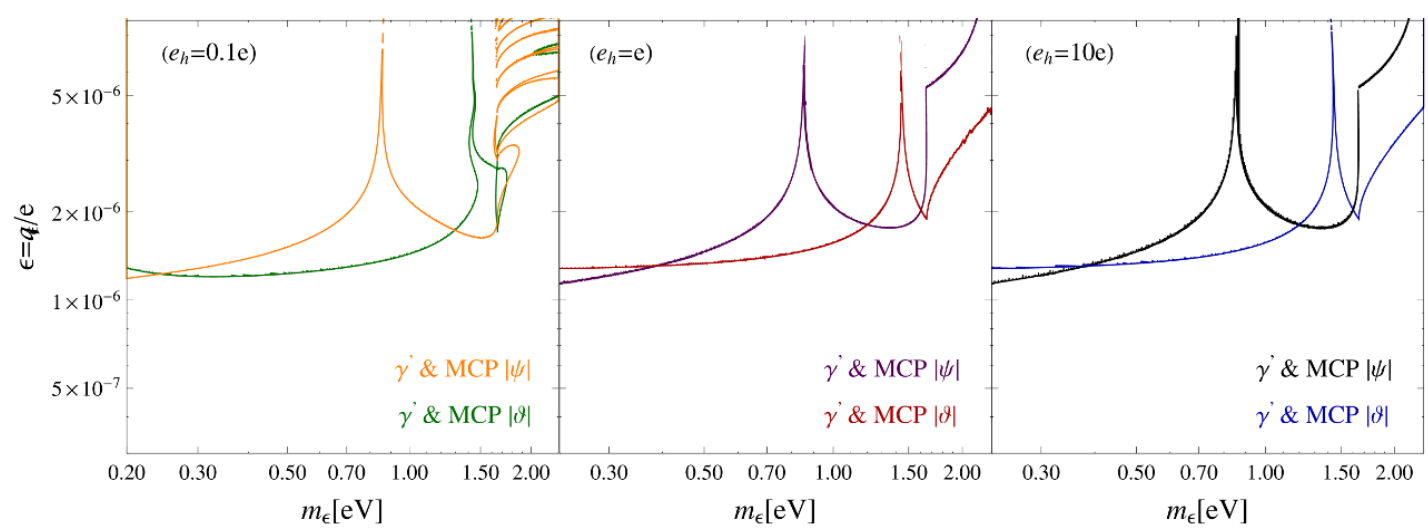

Figure 3. Parameter space to be ruled out for MCPs in a model with paraphotons $\left(\gamma^{\prime}\right)$. The expected exclusion limits have been obtained by assuming the absence of signals in a polarimetric setup assisted by a circularly polarized wave associated with the nanosecond frontend of the PHELIX laser. Here the projected sensitivities on the kinetic mixing parameter for various values of the hidden coupling constant are displayed by contour lines [see legend].

Let us put the projected bounds displayed in figures 2 and 3 into perspective with the constraints deduced from astrophysical and cosmological arguments which are, indeed, stronger [5-8]. For MCPs, the most stringent limits have been set from the absence of anomalous stellar evolutions, presumably induced by the energy loss accompanied by their emissions from the star. When considering this idea in the helium-burning phase of Horizontal-Branch (HB) stars, the coupling strengh turns out to be bounded by [43]

$$
\epsilon \leq 2 \times 10^{-14} \quad \text { for } \quad m_{\epsilon} \lesssim \mathrm{keV} .
$$

Less stringent limits have been found from the solar monitoring of neutrino flux. However, the previous limitations may not be directly applicable to our results because their derivation relies on the assumption that the effective vertices [eq. (2.2)] which allow us to predict phenomenological aspects in laboratory-based experiments, are equally valid in the stellar context, where the momentum transfer $\gtrsim \mathrm{keV}$ is substantially higher than the typical scale associated with lab conditions $\sim \mu \mathrm{eV}[21,45]$. A suitable comparison between the upper bounds resulting from these scenarios would require to take into account the renormalization effects of the coupling constants over the nine orders of magnitude which separate the characteristic energy scales, otherwise notable uncertainties are introduced. The dependence of the coupling strength on the momentum transfer of each production event might attenuate the stellar cooling rate due to MCPs escape, leading to a less severe limit.

On the other hand, the study of the solar emission of hidden massive photons caused by the kinetic mixing currently establishes the most restrictive bounds on the parameter space $\left(\chi, m_{\gamma^{\prime}}\right)$ among the astrophysical arguments. Indeed, for paraphoton masses below $m_{\gamma^{\prime}} \sim 3 \mathrm{eV}$ it has been determined that [88]

$$
\chi<4 \times 10^{-12}\left(\mathrm{eV} / m_{\gamma^{\prime}}\right) .
$$

In contrast to the solar investigation, in the present study we have assumed that the virtuality provided by the polarization tensor is stronger than the mass of the hidden-photon 
field. As one can read off from sections 2.1 and 2.2, this energy regime is characterized by the condition $m_{\gamma^{\prime}} \ll\left(\pi_{ \pm} / \chi\right)^{1 / 2}$. When evaluating this relation with the projected bound resulting from PHELIX $\left[\chi<2 \times 10^{-6}\right.$ at $\left.m_{\epsilon}=m_{1}\right]$, we find that $m_{\gamma^{\prime}} \ll o\left[10^{-1}-1\right] \mu \mathrm{eV}$, for which eq. (3.3) leads to upper bounds less stringent than the values predicted in figures 2 and 3. It is worth observing that, for masses $m_{\gamma^{\prime}} \lesssim 10^{-8} \mathrm{eV}$, the sensitivity limits found for $\chi$ are comparable and even stronger than those resulting from testing the Coulomb law by Cavendish type experiments [25]. Particularly, in the mass sector $10^{-13} \mathrm{eV}<m_{\gamma^{\prime}}<10^{-10} \mathrm{eV}$, the projected bounds are more stringent than those resulting from the Cosmic Microwave Background ${ }^{9}$ and, if the paraphoton mass turns out to be smaller than $\sim 10^{-15} \mathrm{eV}$, then our sensitivity limits might provide the best model independent results on the mixing parameter $\chi$, including the interplay of MCPs with $m_{\epsilon} \sim \mathrm{eV}$.

Besides, the fact that the astrophysical environment differs from the well controlled laboratory conditions opens a portal for various suppression channels that might be active in stellar objects but escape from our perception. Notably, we mention the mechanism involving two paraphotons - one massless and one massive (mass $m_{\gamma^{\prime}}$ ) - minimally coupled to dark fermions with opposite hidden charges [44]. It has been theoretically demonstrated that, for typical values of HB stars, this configuration relaxes the astrophysical constraint to $\epsilon<4 \times 10^{-8}\left([\mathrm{eV}] / m_{\gamma^{\prime}}\right)^{2}$ provided that the "running" coupling strength corresponds to the value given in eq. (3.2). Another vulnerable aspect to take into account is the plausible dependence of the effective vertices on the environmental parameters such as density and temperature of the star, both being higher than in laboratory-based experiments. Investigations in this direction have demonstrated that the astrophysical bounds are extremely sensitive to these macroscopic quantities [45, 86, 87]. Indeed, they might actually suppress the production of MCPs, this way attenuating the resulting limits.

\subsection{Identification of promising scenarios}

So far we have investigated the plausible situation in which no optical change is detected. In this subsection, we shall examine the case where the induced ellipticity and rotation of the outgoing probe beam - due to the vacuum polarization effects of MCPs and a hidden-photon field - become manifest. In first instance, a measurement of the absolute value of the aforementioned observable should be enough for finding the values of $\epsilon$ and $m_{\epsilon}$ provided that only pure MCPs are realized in nature. However, with the inclusion of the hidden-photon field the mixing parameter $\chi$ emerges and consequently, the polarimetric measurements by themselves do not unambiguously determine the unknown particle attributes. This situation might even become worse if other dark matter candidates such as axion-like particles would exist as well at the energy scale relevant for MCPs and paraphotons. Valuable information is however at our disposal: by investigating the signal dependencies on the available experimental quantities such as the intense laser parameter $\xi$, the temporal length $\tau$ and the wave length of the probe beam $\lambda=2 \pi \omega^{-1}$ one can establish the phenomenological differences that result from the models under consideration.

\footnotetext{
${ }^{9} \mathrm{~A}$ summary of astrophysical, cosmological and laboratory constraints for hidden photons, i. e., kinetic mixing vs mass $m_{\gamma^{\prime}}$, can be found in refs. [5-7].
} 


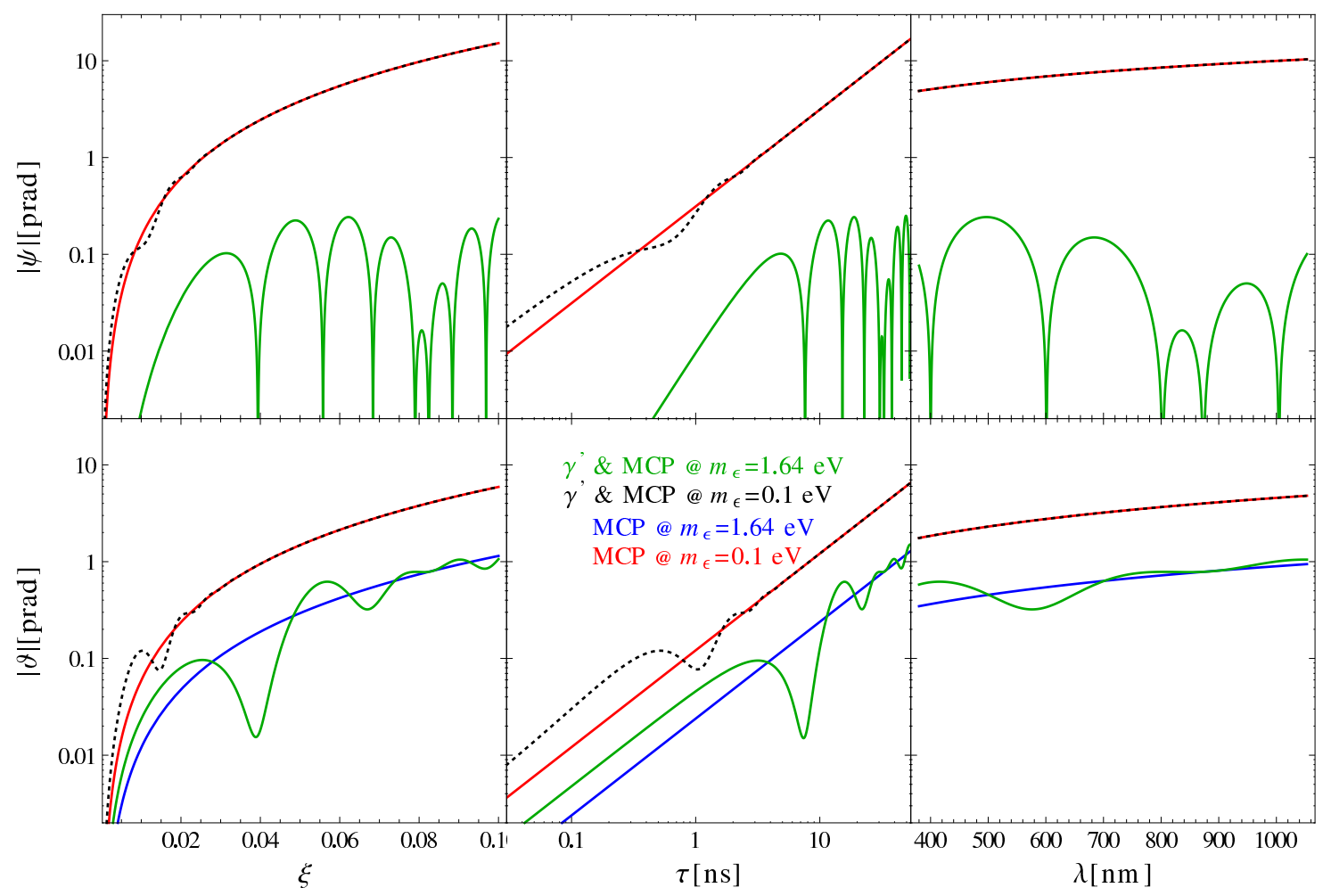

Figure 4. Dependence of the absolute value of the ellipticity $|\psi|$ [upper panels] and rotation angle $|\vartheta|$ [lower panels] on the intensity parameter $\xi$ [left panel], pulse length $\tau$ [central panel] and wavelength of the probe $\lambda$ [right panel]. As a benchmark point we assume a massless hidden photon field with kinetic mixing parameter $\chi=5 \times 10^{-7}$ and hidden coupling $e_{h}=e$. In each plot the remaining external parameters are kept at $\xi=6.4 \times 10^{-2}, \tau=20 \mathrm{~ns}, \boldsymbol{k} \|-\varkappa, \lambda=\lambda_{0} / 2$ with $\lambda_{0}=2 \pi \varkappa_{0}^{-1}=1053 \mathrm{~nm}$ the wavelength of the intense laser field. Here the outcomes resulting from a pure MCP model at $m_{\epsilon}=0.1 \mathrm{eV}$ are shown in red, whereas the respective patterns at the first threshold mass $m_{1} \approx(k \varkappa / 2)^{1 / 2}$ are in blue. The curves in green and dotted black were obtained by including the paraphoton field. They also correspond to the case in which the mass of the minicharges are $m_{\epsilon}=m_{1}$ and $m_{\epsilon}=0.1 \mathrm{eV}$, respectively.

Figure 4 summarizes the behavior of the signals not only when the MCP mass coincides with the first threshold mass $m_{\epsilon}=m_{1} \approx(k \varkappa / 2)^{1 / 2}$ but also at $m_{\epsilon}=0.1 \mathrm{eV}$. The corresponding results associated with the pure $\mathrm{MCP}$ scenario are plotted in blue and red, whereas the outcomes including the effects of a hidden-photon field are shown in green and black dotted curves. All these results were derived by using the benchmark parameters of the nanosecond frontend of PHELIX $\left[\xi=6.4 \times 10^{-2}, \tau=20 \mathrm{~ns}, \lambda_{0}=1053 \mathrm{~nm}\right]$ and by considering a probe beam with $\lambda=\lambda_{0} / 2$ colliding head on with the intense laser wave. Generally speaking we find that both signals tend to grow with the increase of the external laser attributes. At $m_{\epsilon}=0.1 \mathrm{eV}$ and small values of the intensity parameter $\xi<4 \times 10^{-2}$, the dependencies of the ellipticity $|\psi|$ [upper panels] and rotation angle $|\vartheta|$ [lower panels] show slight differences between the pure MCP model and the scenario dealing with the paraphoton effects. Precisely in this region the characteristic time of the transition process $\sim \chi^{2} \kappa_{ \pm}^{-1}$ becomes much bigger than the pulse length $[\tau=20 \mathrm{~ns}]$ and the oscillatory pat- 
terns due to the photon-paraphoton oscillation alters the signal compared to a pure MCPs model. Conversely, for values of $\xi>4 \times 10^{-2}, \chi^{2} \kappa_{ \pm}^{-1}$ turns out to be smaller than $\tau$ leading to exponential suppressions of the hidden-photon effects described in section 2.4. A similar behavior occurs for fixed $\xi=6.4 \times 10^{-2}$ as the pulse length $\tau$ varies. Indeed, the central panel in figure 4 shows that at $m_{\epsilon}=0.1 \mathrm{eV}$ and duration smaller than $\lesssim 6$ ns the signal starts to be sensitive for hidden-photon effects since the characteristic time turns out to be greater than the pulse length of the strong laser wave. Observe that, at $m_{\epsilon}=0.1 \mathrm{eV}$, the respective dependencies of the observables on the wavelength of the probe beam do not reveal any differences. This is because, for the remaining benchmark parameters, the pure MCP contributions turn out to be dominant.

The signal drastically changes at the first threshold mass. Here the minicharges tend to be produced at rest $\left[v_{1} \rightarrow 0\right]$, and the leading order terms of the absorption coefficients [eqs. (2.25) and (2.26)] decrease as $\kappa_{+, 1} \propto v_{1}$ and $\kappa_{-, 1} \propto o\left(v_{1}^{2}\right)$, respectively [57]. Certainly, at this point, the contributions coming from the second threshold, i.e. $\kappa_{ \pm, 2} \propto \xi_{\epsilon}^{4}$ [see eq. (2.29)] might also be important. Whatever be the dominant case, the main outcome would be a noticeable increment in the characteristic times $\chi^{2} \kappa_{ \pm}^{-1}$, which can reach values much larger than the corresponding pulse length $\tau$. In such a situation, the damping factors in eq. (2.43) can be approached by unity, the term associated with the pure MCP model becomes negligible ${ }^{10}$ and the ellipticity follows a fluctuating pattern

$$
|\psi(\tau)| \approx \frac{1}{2} \chi^{2}\left|\cos \left(\frac{n_{+}-1}{\chi^{2}} \omega_{\boldsymbol{k}} \tau\right)-\cos \left(\frac{n_{-}-1}{\chi^{2}} \omega_{\boldsymbol{k}} \tau\right)\right| .
$$

The outcomes displayed in the upper panel of figure 4 clearly highlight this trend. According to eq. (3.4), such a pattern is a direct consequence of the photon-paraphoton transitions, whose oscillation probabilities [eq. (2.38)] reduce to the expressions $\mathcal{P}_{\gamma_{ \pm} \rightarrow \gamma_{ \pm}^{\prime}}(\tau) \approx$ $4 \chi^{2} \sin ^{2}\left(\frac{\mu_{ \pm}^{2}}{4 \omega_{k}} \tau\right)$ with $\mu_{ \pm}^{2}=2 \frac{n_{ \pm}-1}{\chi^{2}} \omega_{k}^{2}$ which resemble the one resulting from the massive paraphoton theory $[11,12]$. Regarding the behavior of the rotation angle [lower panel in figure 4], the situation is slightly different. Based on similar arguments, we found that eq. (2.42) approaches to

$$
|\vartheta(\tau)| \approx \frac{1}{2}\left|\left(n_{+}-n_{-}\right) \omega_{\boldsymbol{k}} \tau+\chi^{2}\left[\sin \left(\frac{n_{+}-1}{\chi^{2}} \omega_{\boldsymbol{k}} \tau\right)-\sin \left(\frac{n_{-}-1}{\chi^{2}} \omega_{\boldsymbol{k}} \tau\right)\right]\right| .
$$

Here the occurrence of fluctuations is also assignable to the photon-paraphoton oscillations. However, in contrast to our previous analysis, the standard result for a model without paraphoton - first term in the above equation - remains important and even dominant as the external parameters increase.

In figure 5, we plot the ellipticity and rotation of the polarization plane with respect to the unknown parameters of the theory. Particularly, the left and right panels reveal how the signals might change with the mixing parameter $\chi$ and the relative hidden coupling $e_{h} / e$. From the former we note that at $m_{\epsilon}=0.1 \mathrm{eV}$ the theory including a hidden-photon field follows the path dictated by the pure MCPs model. Besides, a fast decrease in both

\footnotetext{
${ }^{10}$ This is why the blue curve does not appear in the upper panels associated with the ellipticity.
} 


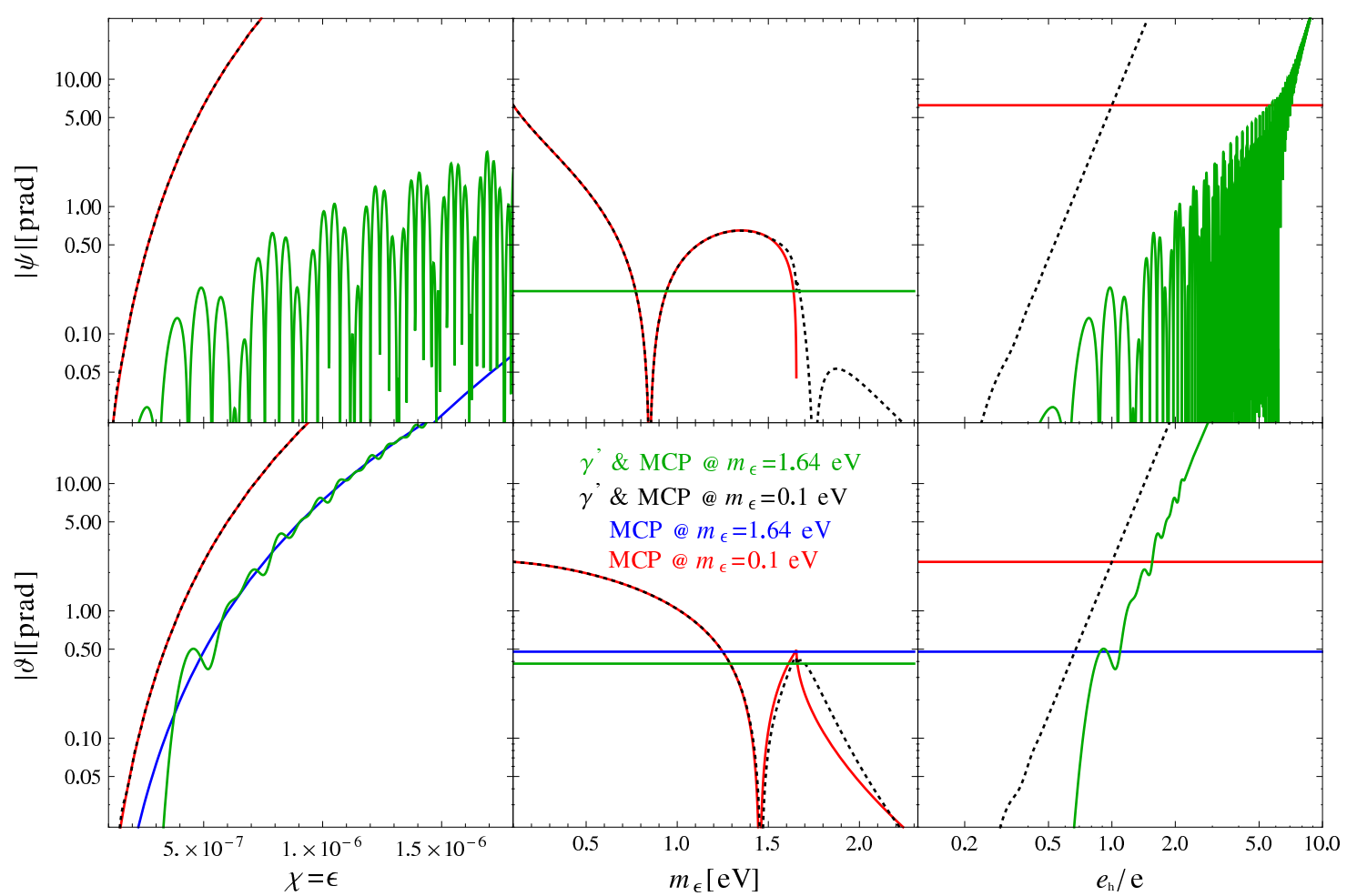

Figure 5. Dependence of the absolute value of the ellipticity $|\psi|$ [upper panels] and rotation angle $|\vartheta|$ [lower panels] on the kinetic mixing parameter $\chi$ [left panel], mass $m_{\epsilon}$ [central panel] and the relative hidden coupling $e_{h} / e$ [right panel]. The same benchmark values of figure $4-\xi=6.4 \times 10^{-2}$, $\tau=20 \mathrm{~ns}, \boldsymbol{k} \|-\varkappa, \lambda=\lambda_{0} / 2, \lambda_{0}=2 \pi \varkappa_{0}^{-1}=1053 \mathrm{~nm}$ - have been used.

signals can be observed for small values of $\chi$. This trend is also manifest with respect to $e_{h} / e$ at the same reference mass [black dotted curve]. Here, the outcome resulting from the pure MCP scenario [horizontal red and blue lines] are not sensitive to variations of the relative hidden coupling because the latter only arises within the framework of a hiddenphoton model. In both - left and right - panels, one recognizes the fluctuating patterns [eqs. (3.4) and (3.5)] induced by the photon-paraphoton oscillations at the first threshold mass $m_{1}=1.64 \mathrm{eV}$. It can be seen that the oscillations caused by the photon-paraphoton coupling tend to be less pronounced as $e_{h} / e$ increases. The central panel of this figure displays how both observables depend on the mass $m_{\epsilon}$ of MCPs. There, in blue and green lines are indicated the reference values obtained for a fixed mass $m_{\epsilon}=m_{1}$. The ellipticity resulting from this scenario clearly shows the discontinuity at the first threshold mass discussed in section 2.3 and associated with the nature of the absorption coefficients. This feature is smoothed when a hidden photon field is considered [see dotted black curve]. At the first threshold, this observable is constant in both scenarios, with the particularity of being extremely tiny in the pure MCPs model [see footnote 10]. Conversely, the dependence of $|\vartheta(\tau)|$ with respect to the mass $m_{\epsilon}$ follows a continuous path in both contexts and only slight differences appear for masses above the first threshold mass $m_{1}$. This last observation could be anticipated based on the analyses of our previous discussions. 


\section{Conclusions}

Accurate polarimetric techniques, searching for the birefringence and dichroism of the quantum vacuum polarized by the field of a laser pulse, can be powerful probes for testing some effective theories beyond SM. We have considered the particular situation in which the external laser wave is circularly polarized and extended the results derived in ref. [57] by incorporating the effects induced by paraphotons. In order to polarimetrically verify the realization of the considered models it is essential to gain detailed information on their respective phenomenologies. As such, one of the main goals of this work was to provide features which can allow us to distinguish between the pure MCPs scenario and the coexistence of a hidden-photon field. Throughout, we noted that the possibility of exchanging photons with the external wave renders the description of the problem more cumbersome than in the case of a static magnetic field. These nontrivial properties, in conjunction with the energy-momentum balance lead to the appearance of thresholds closely associated with a hypothetical photo-production of pairs of MCPs, their masses being determined by the frequencies of both laser fields. For $\xi_{\epsilon}<1$ and near the first threshold, the chiral activity of the "medium" turns out to be quite pronounced and the searches of very light MCPs and hidden-photons by using polarimetric setups appear promising. In connection, the induced ellipticity and rotation of the polarization plane of the probe beam were determined. When evaluating such observables with the attributes of modern laser systems, stringent sensitivity limits on the parameter spaces were found under the assumption of no signal detection. These first estimates reveal that a laser wave with a long temporal length and a moderate intensity might be an external-field source suitable for searching very light weakly interacting particles with masses in the $\mathrm{eV}$ range. As such our outcomes agree with and complement the results obtained in a previous investigation developed within the context of axion-like particles [58].

\section{Acknowledgments}

We gratefully acknowledge funding by the German Research Foundation (DFG) under Grant No. MU 3149/2-1 during the final phase of this study.

Open Access. This article is distributed under the terms of the Creative Commons Attribution License (CC-BY 4.0), which permits any use, distribution and reproduction in any medium, provided the original author(s) and source are credited.

\section{References}

[1] E. Witten, Some Properties of O(32) Superstrings, Phys. Lett. B 149 (1984) 351 [InSPIRE].

[2] O. Lebedev, H.P. Nilles, S. Ramos-Sanchez, M. Ratz and P.K.S. Vaudrevange, Heterotic mini-landscape. (II). Completing the search for MSSM vacua in a Z(6) orbifold, Phys. Lett. B 668 (2008) 331 [arXiv:0807.4384] [INSPIRE].

[3] O. Lebedev and S. Ramos-Sanchez, The NMSSM and String Theory, Phys. Lett. B 684 (2010) 48 [arXiv:0912.0477] [InSPIRE]. 
[4] M. Goodsell, J. Jaeckel, J. Redondo and A. Ringwald, Naturally Light Hidden Photons in LARGE Volume String Compactifications, JHEP 11 (2009) 027 [arXiv:0909.0515] [INSPIRE].

[5] J. Jaeckel and A. Ringwald, The Low-Energy Frontier of Particle Physics, Ann. Rev. Nucl. Part. Sci. 60 (2010) 405 [arXiv: 1002.0329] [InSPIRE].

[6] A. Ringwald, Exploring the Role of Axions and Other WISPs in the Dark Universe, Phys. Dark Univ. 1 (2012) 116 [arXiv:1210.5081] [INSPIRE].

[7] J.L. Hewett et al., Fundamental Physics at the Intensity Frontier, Proceedings of the 2011 workshop on Fundamental Physics at the Intensity Frontier, [arXiv:1205.2671] [INSPIRE].

[8] R. Essig, J.A. Jaros, W. Wester, P.H. Adrian, S. Andreas et al., Working Group Report: new light weakly coupled particles, arXiv:1311.0029 [INSPIRE].

[9] L.B. Okun, Limits of electrodynamics: paraphotons?, Sov. Phys. JETP 56 (1982) 502 [Zh. Eksp. Teor. Fiz. 83 (1982) 892] [INSPIRE].

[10] P. Langacker, The Physics of Heavy Z' Gauge Bosons, Rev. Mod. Phys. 81 (2009) 1199 [arXiv:0801.1345] [INSPIRE].

[11] M. Ahlers, H. Gies, J. Jaeckel, J. Redondo and A. Ringwald, Light from the hidden sector, Phys. Rev. D 76 (2007) 115005 [arXiv:0706.2836] [INSPIRE].

[12] M. Ahlers, H. Gies, J. Jaeckel, J. Redondo and A. Ringwald, Laser experiments explore the hidden sector, Phys. Rev. D 77 (2008) 095001 [arXiv:0711.4991] [INSPIRE].

[13] B. Holdom, Two U(1)'s and $\epsilon$ charge shifts, Phys. Lett. B 166 (1986) 196 [INSPIRE].

[14] B.A. Dobrescu and I. Mocioiu, Spin-dependent macroscopic forces from new particle exchange, JHEP 11 (2006) 005 [hep-ph/0605342] [INSPIRE].

[15] H. Gies, J. Jaeckel and A. Ringwald, Polarized light propagating in a magnetic field as a probe of millicharged fermions, Phys. Rev. Lett. 97 (2006) 140402 [hep-ph/0607118] [INSPIRE].

[16] M. Ahlers, H. Gies, J. Jaeckel and A. Ringwald, On the particle interpretation of the PVLAS data: neutral versus charged particles, Phys. Rev. D 75 (2007) 035011 [hep-ph/0612098] [INSPIRE].

[17] B. Batell and T. Gherghetta, Localized U(1) gauge fields, millicharged particles and holography, Phys. Rev. D 73 (2006) 045016 [hep-ph/0512356] [INSPIRE].

[18] F. Brummer, J. Jaeckel and V.V. Khoze, Magnetic mixing: electric minicharges from magnetic monopoles, JHEP 06 (2009) 037 [arXiv: 0905. 0633] [INSPIRE].

[19] E. Dudas, Y. Mambrini, S. Pokorski and A. Romagnoni, Extra U(1) as natural source of a monochromatic gamma ray line, JHEP 10 (2012) 123 [arXiv:1205.1520] [INSPIRE].

[20] B. Döbrich and H. Gies, Interferometry of light propagation in pulsed fields, Europhys. Lett. 87 (2009) 21002 [arXiv:0904.0216] [INSPIRE].

[21] H. Gies, External fields as a probe for fundamental physics, J. Phys. A 41 (2008) 164039 [arXiv: 0711.1337] [INSPIRE].

[22] W. Dittrich and H. Gies, Probing the quantum vacuum, Springer, Heidelberg Germany (2000).

[23] K. Hattori and K. Itakura, Vacuum birefringence in strong magnetic fields: (I) photon polarization tensor with all the Landau levels, Ann. Phys. 330 (2013) 23 [arXiv:1209.2663] [INSPIRE]. 
[24] S. Villalba-Chávez and A.E. Shabad, QED with external field: Hamiltonian treatment for anisotropic medium formed by the Lorentz-non-invariant vacuum, Phys. Rev. D 86 (2012) 105040 [arXiv:1206.4491] [INSPIRE].

[25] J. Jaeckel, Probing minicharged particles with tests of Coulomb's law, Phys. Rev. Lett. 103 (2009) 080402 [arXiv:0904.1547] [INSPIRE].

[26] J. Jaeckel and S. Roy, Spectroscopy as a test of Coulomb's law: a probe of the hidden sector, Phys. Rev. D 82 (2010) 125020 [arXiv: 1008.3536] [InSPIRE].

[27] J. Redondo and A. Ringwald, Light shining through walls, Contemp. Phys. 52 (2011) 211 [arXiv: 1011.3741] [INSPIRE].

[28] P. Arias, J. Jaeckel, J. Redondo and A. Ringwald, Optimizing light-shining-through-a-wall experiments for axion and other WISP searches, Phys. Rev. D 82 (2010) 115018 [arXiv: 1009.4875] [INSPIRE].

[29] R. Cameron, G. Cantatore, A.C. Melissinos, G. Ruoso, Y. Semertzidis et al., Search for nearly massless, weakly coupled particles by optical techniques, Phys. Rev. D 47 (1993) 3707 [INSPIRE].

[30] PVLAS collaboration, E. Zavattini et al., New PVLAS results and limits on magnetically induced optical rotation and ellipticity in vacuum, Phys. Rev. D 77 (2008) 032006 [arXiv:0706.3419] [INSPIRE].

[31] PVLAS collaboration, F. Della Valle et al., Measurements of vacuum magnetic birefringence using permanent dipole magnets: the PVLAS experiment, New J. Phys. 15 (2013) 053026 [arXiv: 1301.4918] [INSPIRE].

[32] R. Battesti et al., The BMV experiment: a novel apparatus to study the propagation of light in a transverse magnetic field, Eur. Phys. J. D 46 (2008) 323.

[33] S.-J. Chen, H.-H. Mei and W.-T. Ni, Q $\mathscr{B}$ A experiment to search for vacuum dichroism, pseudoscalar-photon interaction and millicharged fermions,

Mod. Phys. Lett. A 22 (2007) 2815 [hep-ex/0611050] [INSPIRE].

[34] ALPS collaboration, K. Ehret et al., New ALPS Results on Hidden-Sector Lightweights, Phys. Lett. B 689 (2010) 149 [arXiv:1004.1313] [INSPIRE].

[35] ALPS collaboration, K. Ehret et al., Resonant laser power build-up in ALPS: a 'light-shining-through-walls' experiment, Nucl. Instrum. Meth. A 612 (2009) 83 [arXiv:0905.4159] [INSPIRE].

[36] GammeV (T-969) collaboration, A.S. Chou et al., Search for axion-like particles using a variable baseline photon regeneration technique, Phys. Rev. Lett. 100 (2008) 080402 [arXiv:0710.3783] [INSPIRE].

[37] J.H. Steffen and A. Upadhye, The GammeV suite of experimental searches for axion-like particles, Mod. Phys. Lett. A 24 (2009) 2053 [arXiv:0908.1529] [INSPIRE].

[38] A. Afanasev, O.K. Baker, K.B. Beard, G. Biallas, J. Boyce et al., New experimental limit on optical photon coupling to neutral, scalar bosons, Phys. Rev. Lett. 101 (2008) 120401 [arXiv:0806.2631] [INSPIRE].

[39] A.V. Afanasev, O.K. Baker and K.W. McFarlane, Production and detection of very light spin-zero bosons at optical frequencies, hep-ph/0605250 [INSPIRE]. 
[40] OSQAR collaboration, P. Pugnat et al., First results from the OSQAR photon regeneration experiment: no light shining through a wall, Phys. Rev. D 78 (2008) 092003

[arXiv:0712.3362] [INSPIRE].

[41] C. Robilliard et al., No light shining through a wall, Phys. Rev. Lett. 99 (2007) 190403 [arXiv:0707.1296] [INSPIRE].

[42] M. Fouche et al., Search for photon oscillations into massive particles, Phys. Rev. D 78 (2008) 032013 [arXiv:0808.2800] [InSPIRE].

[43] S. Davidson, S. Hannestad and G. Raffelt, Updated bounds on millicharged particles, JHEP 05 (2000) 003 [hep-ph/0001179] [INSPIRE].

[44] E. Masso and J. Redondo, Compatibility of CAST search with axion-like interpretation of PVLAS results, Phys. Rev. Lett. 97 (2006) 151802 [hep-ph/0606163] [INSPIRE].

[45] J. Jaeckel, E. Masso, J. Redondo, A. Ringwald and F. Takahashi, The need for purely laboratory-based axion-like particle searches, Phys. Rev. D 75 (2007) 013004 [hep-ph/0610203] [INSPIRE].

[46] R. Bähre et al., Any light particle search II - Technical Design Report, 2013 JINST 8 T09001 [arXiv:1302.5647] [INSPIRE].

[47] P. Sikivie, D.B. Tanner and K. van Bibber, Resonantly enhanced axion-photon regeneration, Phys. Rev. Lett. 98 (2007) 172002 [hep-ph/0701198] [INSPIRE].

[48] G. Müller, P. Sikivie, D.B. Tanner and K. van Bibber, Detailed design of a resonantly-enhanced axion-photon regeneration experiment, Phys. Rev. D 80 (2009) 072004 [arXiv: 0907.5387] [INSPIRE].

[49] H. Gies, J. Jaeckel and A. Ringwald, Accelerator Cavities as a Probe of Millicharged Particles, Europhys. Lett. 76 (2006) 794 [hep-ph/0608238] [INSPIRE].

[50] B. Döbrich, H. Gies, N. Neitz and F. Karbstein, Magnetically amplified tunneling of the 3rd kind as a probe of minicharged particles, Phys. Rev. Lett. 109 (2012) 131802 [arXiv: 1203.2533] [INSPIRE].

[51] B. Döbrich, H. Gies, N. Neitz and F. Karbstein, Magnetically amplified light-shining-through-walls via virtual minicharged particles, Phys. Rev. D 87 (2013) 025022 [arXiv: 1203.4986] [INSPIRE].

[52] H. Gies, Strong laser fields as a probe for fundamental physics, Eur. Phys. J. D 55 (2009) 311 [arXiv:0812.0668] [InSPIRE].

[53] B. Döbrich and H. Gies, Axion-like-particle search with high-intensity lasers, JHEP 10 (2010) 022 [arXiv: 1006.5579] [INSPIRE].

[54] B. Döbrich and H. Gies, High-Intensity Probes of Axion-Like Particles, arXiv:1010.6161 [INSPIRE].

[55] D. Tommasini, A. Ferrando, H. Michinel and M. Seco, Precision tests of QED and non-standard models by searching photon-photon scattering in vacuum with high power lasers, JHEP 11 (2009) 043 [arXiv:0909.4663] [INSPIRE].

[56] S. Villalba-Chávez and A. Di Piazza, Axion-induced birefringence effects in laser driven nonlinear vacuum interaction, JHEP 11 (2013) 136 [arXiv:1307.7935] [INSPIRE]. 
[57] S. Villalba-Chávez and C. Müller, Searching for minicharged particles via birefringence, dichroism and Raman spectroscopy of the vacuum polarized by a high-intensity laser wave, Annals Phys. 339 (2013) 460 [arXiv:1306.6456] [INSPIRE].

[58] S. Villalba-Chávez, Laser-driven search of axion-like particles including vacuum polarization effects, Nucl. Phys. B 881 (2014) 391 [arXiv:1308.4033] [INSPIRE].

[59] http://www.eli-beams.eu/.

[60] http://www.xcels.iapras.ru/.

[61] T. Heinzl, B. Leifeld, K. U. Amthor, H. Schwoerer, R. Sauerbrey and A. Wipf, On the observation of vacuum birefringence, Opt. Comm. 267 (2006) 318 [hep-ph/0601076] [INSPIRE].

[62] B. King, P. Böhl and H. Ruhl, Interaction of photons traversing a slowly varying electromagnetic background, Phys. Rev. D 90 (2014) 065018 [arXiv:1406.4139] [INSPIRE].

[63] A. Di Piazza, A.I. Milstein and C.H. Keitel, Photon splitting in a laser field, Phys. Rev. A 76 (2007) 032103 [arXiv:0704.0695] [InSPIRE].

[64] B. King, A. Di Piazza and C.H. Keitel, A matterless double slit, Nature Photon. 4 (2010) 92 [arXiv: 1301.7038] [INSPIRE].

[65] K.Z. Hatsagortsyan and G.Y. Kryuchkyan, Bragg scattering of light in vacuum structured by strong periodic fields, Phys. Rev. Lett. 107 (2011) 053604.

[66] A. Di Piazza, C. Müller, K.Z. Hatsagortsyan and C.H. Keitel, Extremely high-intensity laser interactions with fundamental quantum systems, Rev. Mod. Phys. 84 (2012) 1177 [arXiv:1111.3886] [INSPIRE].

[67] J.S. Schwinger, On gauge invariance and vacuum polarization, Phys. Rev. 82 (1951) 664 [INSPIRE].

[68] F. Hebenstreit, R. Alkofer, G.V. Dunne and H. Gies, Momentum signatures for Schwinger pair production in short laser pulses with sub-cycle structure, Phys. Rev. Lett. 102 (2009) 150404 [arXiv:0901.2631] [INSPIRE].

[69] I. Akal, S. Villalba-Chávez and C. Müller, Electron-positron pair production in a bifrequent oscillating electric field, Phys. Rev. D 90 (2014) 113004 [arXiv: 1409.1806] [INSPIRE].

[70] https://www.gsi.de/en/start/research/forschungsgebiete_und_experimente/ appa_pni_gesundheit/plasma_physicsphelix/phelix.htm.

[71] C. Labaune et al., Fusion reactions initiated by laser-accelerated particle beams in a laser-produced plasma, Nat. Commun. 4 (2013) 2506 [arXiv:1310.2002] [INSPIRE].

[72] R.J. Rivers, Path integral methods in Quantum field theory, Cambridge University Press, U.S.A. (1987).

[73] S. Villalba-Chávez, R. Alkofer and K. Schwenzer, On the connection between Hamilton and Lagrange formalism in Quantum Field Theory, J. Phys. G 37 (2010) 085003 [arXiv: 0807.2146] [INSPIRE].

[74] E.S. Fradkin, Quantum Field Theory and Hydrodynamics, Proceedings (Trudy) of the P.N. Lebedev Physics Institute. Vol. 29, Consultants Bureau, New York U.S.A. (1967).

[75] R. Alkofer and L. von Smekal, The Infrared behavior of QCD Green's functions: Confinement dynamical symmetry breaking and hadrons as relativistic bound states, Phys. Rept. 353 (2001) 281 [hep-ph/0007355] [INSPIRE]. 
[76] V.N. Bă̌er, A.I. Mil'shteĭn and V.M. Strakhovenko, Interaction between a photon and an intense electromagnetic wave, Sov. Phys. JETP 42 (1976) 961 [Zh. Eksp. Teo. Fiz. 69 (1975) 1893].

[77] I. Affleck, Photon propagation in a plane wave field, J. Phys. A 21 (1988) 693 [InSPIRE].

[78] V. Dinu, T. Heinzl, A. Ilderton, M. Marklund and G. Torgrimsson, Vacuum refractive indices and helicity flip in strong-field QED, Phys. Rev. D 89 (2014) 125003 [arXiv:1312.6419] [INSPIRE].

[79] S. Villalba-Chávez and C. Müller, Photo-production of scalar particles in the field of a circularly polarized laser beam, Phys. Lett. B 718 (2013) 992 [arXiv:1208.3595] [INSPIRE].

[80] W. Greiner and J. Reinhardt, Quantum Electrodynamics, Springer, Berlin Heidelberg (2009).

[81] I.S. Gradshteyn and I.M. Ryzhik, Table of Integrals, Series and Products, seventh edition, Elsevier, San Diego (2007).

[82] V.N. Baĭer, V.M. Katkov and V.M. Strakhovenko. Electromagnetic processes at high energies in oriented single crystals, World Scientific, Singapore (1998).

[83] W. Becker and H. Mitter, Vacuum polarization in laser fields, J. Phys. A 8 (1975) 1638.

[84] K. Muroo, N. Ninomiya, M. Yoshino and Y. Takubo Measurement of the Cotton-Mouton constants of noble atoms, J. Opt. Soc. Am. B 20 (2003) 2249.

[85] http://www.hzdr.de/db/Cms?pNid=427\&pOid=35325.

[86] J. Jaeckel, E. Masso, J. Redondo, A. Ringwald and F. Takahashi, We need lab experiments to look for axion-like particles, hep-ph/0605313 [INSPIRE].

[87] E. Masso and J. Redondo, Evading astrophysical constraints on axion-like particles, JCAP 09 (2005) 015 [hep-ph/0504202] [INSPIRE].

[88] J. Redondo and G. Raffelt, Solar constraints on hidden photons re-visited, JCAP 08 (2013) 034 [arXiv: 1305.2920] [INSPIRE]. 